\title{
FORUM
}

\section{Postkoloniale Studien und Politikwissenschaft: Komplementäre Defizite, Stand der Forschung und Perspektiven}

\author{
Aram Ziai
}

Postcolonial Studies and Political Science: Complementary Deficits, State of the Art, and Research Perspectives

Abstract: The article deals with the relationship between postcolonial studies and political science. As political science has hitherto largely ignored postcolonial inquiries and perspectives, and postcolonial studies on the other hand have treated political institutions and processes often in a rather superficial manner, the article argues that connecting these fields will prove to be productive and insightful. Based on a survey on the state of the art of political science informed by postcolonialism, a research programme is sketched which operationalises the concepts of orientalism and othering, subalternity and representation/articulation, hybridity and provincialising Europe.

Keywords: Political Science, Postcolonial Studies, Eurocentrism

Schlagwörter: Politikwissenschaft, Postkoloniale Studien, Eurozentrismus

\section{Einleitung}

Die ursprünglich aus der angelsächsischen Literaturwissenschaft stammenden Postkolonialen Studien werden in zunehmendem Maße auch in den Gesellschaftswissenschaften rezipiert, mit der üblichen Verspätung auch hierzulande. Ihr gemeinsamer inhaltlicher Nenner ist, grob vereinfacht, die Thematisierung der Nachwirkungen kolonialer Herrschaft v. a. im Hinblick auf Repräsentationen und Identitäten. Das Adjektiv „postkolonial“ bezieht sich in diesem Kontext auf einen bestimmten theoretischen Ansatz und beinhaltet demnach nicht die Gesamtheit von Arbeiten, die sich mit nachkolonialen Gesellschaften befassen. Der vorliegende Artikel ${ }^{1}$ befasst sich mit dem Verhältnis von Postkolonialen Studien und Politikwissenschaft und plädiert für einen verstärkten Dialog zwischen beiden Forschungstraditionen. Zunächst ist es notwendig zu klären, was genau mit Postkolonialen Studien gemeint ist (Abschnitt 2). Anschließend möchte ich anhand von Beispielen die These illustrieren, dass Postkoloniale Studien und Politikwissenschaft von komplementären Defiziten geprägt sind: politikwissenschaftliche Arbeiten weisen oft wenig Sensibilität für postkoloniale Fragestellungen auf, wäh-

1 Für nützliche Hinweise danke ich den anonymen GutachterInnen sowie der PVS-Redaktion. 
rend die Auseinandersetzung mit politischen Institutionen und Prozessen in postkolonialen Arbeiten oft eher oberflächlich und unsystematisch ist (Abschnitt 3). Im nächsten Abschnitt möchte ich auf den Forschungsstand einer diese Defizite behebenden Postkolonialen Politikforschung eingehen (Abschnitt 4), um anschließend ein daran anknüpfendes Forschungsprogramm vorzustellen, das auf der Operationalisierung zentraler postkolonialer Konzepte beruht, die auf unterschiedliche Bereiche der Politikwissenschaft angewandt werden können (Abschnitt 5).

\section{Postkoloniale Studien}

Im angelsächsischen Raum haben sich die Postcolonial Studies im Laufe der 1990er-Jahre als eigenständiges interdisziplinäres Forschungsfeld durchgesetzt. Wie kaum eine andere Disziplin sind die Postkolonialen Studien eng verknüpft mit spezifischen AutorInnen, die viel zu ihrer Verbreitung beigetragen haben und als ihre Ikonen angesehen werden: Edward Said, Gayatri Spivak und Homi Bhabha haben mit ihren wichtigen Publikationen (Saids Monografie Orientalism [(1978) 1981; siehe auch Said (1993) 1994], Spivaks Artikel Can the Subaltern speak? [(1988) 2008; siehe auch Spivak 1990, 1996] und Bhabhas Aufsätze in Nation and Narration [1990] und The Location of Culture [1994]) das Forschungsfeld nachhaltig geprägt. Das Innovative an ihrem Werk ist, dass sie die Auswirkungen des Kolonialismus v. a. auf der Ebene von Repräsentationen und Identitäten untersuchen - und zu dem Ergebnis kommen, dass diese auch lange nach der formellen Dekolonisierung vielerorts präsent und wirkmächtig sind. $\mathrm{Zu}$ Recht ist darauf hingewiesen worden, dass wichtige Elemente der postkolonialen Theorien auch schon in Ansätzen antikolonialer Intellektueller wie Aimé Césaire, Frantz Fanon oder Mohandas K. Gandhi auffindbar sind, ohne dass dieser Hinweis jedoch den Stellenwert von Said, Spivak und Bhabha spürbar verringert hätte. Dirlik (1994) bemerkt dazu, dass die Etablierung der Postkolonialen Studien demnach maßgeblich mit der Ankunft von „Drittweltintellektuellen“ in den Universitäten des Nordens zusammenhängt, die allerdings von den materiellen Realitäten und sozialen Kämpfen der Dritten Welt abgekoppelt seien. Shohat (1992) vertritt in diesem Kontext die These, dass die Konjunktur des PostkolonialismusKonzepts in den 1990er-Jahren mit der Krise anderer, marxistisch orientienter Konzepte einherging und Vokabeln wie „neo-kolonial“ oder „imperialistisch“ aus den Analysen verdrängte. Beide Thesen werden bei der Abgrenzung des Forschungsfelds noch eine Rolle spielen.

Wichtig in diesem Zusammenhang ist auch, dass alle drei genannten AutorInnen ursprünglich LiteraturwissenschaftlerInnen sind, auch wenn sie interdisziplinär vorgegangen sind und literatur- und sprachwissenschaftliche, historische, psychologische und sozialwissenschaftliche Fragestellungen miteinander verknüpft haben. Andere, ebenfalls einflussreiche VertreterInnen der Postkolonialen Studien entstammen bisweilen auch anderen Fächern wie der Geschichtswissenschaft (Robert Young, Dipesh Chakrabarty), Anthropologie (Ann Laura Stoler, Fernando Coronil, Jean Comaroff) oder der Soziologie (Stuart Hall, Anibal Quijano). PolitikwissenschaftlerInnen sind so gut wie keine zu finden (einzige Ausnahme ist Ti- 
mothy Mitchell). Diese unterschiedlichen disziplinären Ursprünge hängen z. T. auch mit den Kontroversen um die Abgrenzung des Forschungsfelds zusammen, auf die noch einzugehen sein wird.

Mittlerweile existiert eine große Anzahl an Überblicksdarstellungen und Einführungen im Bereich der Postkolonialen Studien (Williams u. Chrisman 1994; Ashcroft et al. 1995; Loomba 1998; Gandhi 1998; Darby 2000; Young 2001, 2003; Mbembe 2001; Grovogui 2007; Conrad u. Shanderia 2002; Castro Varela u. Dhawan 2005 - um nur die wahrscheinlich bekanntesten zu nennen). Hinsichtlich einer Definition des Gegenstandsbereichs der Postkolonialen Studien ist festzustellen, dass über die allgemeine Befassung mit dem Phänomen des Kolonialismus und seinen Auswirkungen hinaus keine Einigkeit über eine präzise Abgrenzung des Forschungsfelds besteht - lediglich über seine oft erwähnte Heterogenität (Ashcroft et al. 1995, S. xv). Ashcroft, Griffiths und Tiffin beziehen sich primär auf „discursive practices“ und „cultural strategies“, definieren aber den Gegenstandsbereich als „the totality of practices [...] which characterise the societies of the post-colonial world from the moment of colonisation to the present day" (1995, S. xv). Williams und Chrisman verstehen post-koloniale Theorie enger gefasst als „critique [...] of the process of production of knowledge about the other" (1994, S. 8). Mit einem ähnlichen Fokus argumentiert Young: "Postcolonial cultural analysis has been concerned with the elaboration of theoretical structures that contest the previous dominant western way of seeing things. [...] ,postcolonial theory' involves a conceptual reorientation towards the perspectives of knowledges, as well as needs, developed outside the west" (2003, S. 4, 6). Loomba wiederum will das Feld über die Auseinandersetzung mit Strukturen der Wissensproduktion erweitern und sieht das Charakteristikum postkolonialer Ansätze in der "contestation of colonial domination and the legacies of colonialism“ (1998, S. 12). Castro Varela und Dhawan pflichten ihr bei: „Postkoloniale Theorie untersucht [...] sowohl den Prozess der Kolonisierung als auch der einer fortwährenden Dekolonisierung und Rekolonisierung. Die Perspektive auf den (Neo) Kolonialismus beschränkt sich dabei nicht auf eine brutale militärische Besetzung und Ausplünderung geographischer Territorien, sondern umfasst auch die Produktion epistemischer Gewalt" (2005, S. 8). Und auch Conrad und Randeria gebrauchen eine weite Definition: „Das Anliegen postkolonialer Ansätze besteht $[\ldots]$ in der Thematisierung des Fortbestehens und Nachwirkens einer Vielzahl von Beziehungsmustern und Effekten kolonialer Herrschaft" (2002, S. 24).

Einig sind sich die Ansätze darin, dass der Kolonialismus aufgrund seiner Nachwirkungen auch nach der Dekolonisierung als unabgeschlossenes Phänomen $\mathrm{zu}$ betrachten ist. Kontrovers ist jedoch die Frage, ob der Gegenstandsbereich Postkolonialer Studien auf der Ebene kolonialer Wissensproduktion zu verorten ist oder ganz allgemein die Effekte kolonialer Herrschaft und somit auch eine Vielzahl von materiellen Praktiken umfasst. In den zitierten Studien scheint sich ein Übergewicht zugunsten der weiten Definition abzuzeichnen. Dies ist insofern erstaunlich, als dass sich dies in der Forschungspraxis in keiner Weise widerspiegelt: Die übergroße Mehrheit der Arbeiten im Bereich der Postkolonialen Studien bezieht sich auf Wissensproduktion, Repräsentationen und Identitäten und klammert materielle Praktiken weitestgehend aus. Hier manifestieren sich auch die dis- 
ziplinären Ursprünge des eigentlich interdisziplinären Ansatzes, seine literaturwissenschaftlichen Wurzeln. Erklären lässt sich dieses Missverhältnis zwischen der oftmals weiten, also auch die Gegenstandsbereiche von Politik- und Wirtschaftswissenschaft umfassenden Definition des Forschungsfelds, und der meist auf die Ebene von Diskursen beschränkten Forschungspraxis mit einer theoriepolitischen Entscheidung angesichts der oben erwähnten Vorwürfe von Shohat (1992) und Dirlik (1994). Um also der Kritik zu begegnen, Postkoloniale Studien seien ein von den eigentlichen Problemen der Dritten Welt losgelöstes, akademisches und daher politisch harmloses Unterfangen, betonen viele ihrer VertreterInnen aus einer kritischen wissenschaftstheoretischen Haltung heraus, dass es ihnen sehr wohl auch um die materiellen Praktiken gehe.

Dazu sind zwei Punkte zu sagen. Zum einen ändert die Entscheidung für eine weite Definition nichts daran, dass sie bisher oftmals ein uneingelöstes Versprechen bleibt: In den meisten Publikationen der Postkolonialen Studien findet eine ernsthafte Auseinandersetzung mit politischen und ökonomischen Strukturen nur am Rande statt (auf die Ausnahmen wird im weiteren Verlauf des Artikels einzugehen sein). Gerade im Hinblick auf die internationale politische Ökonomie ist festzustellen, dass zwar gelegentlich auf eine internationale Arbeitsteilung oder (angeblich) ungerechte Weltmarktstrukturen verwiesen wird, eine nähere Kenntnis beispielsweise selbst der grundlegenden Prinzipien und Abkommen der Welthandelsorganisation jedoch bereits Seltenheitswert hat. Auch Stuart Hall, der in einem vielzitierten Aufsatz die meisten der an den Postkolonialen Studien geübten Kritiken fundiert zu entkräften vermag, gibt den KritikerInnen in diesem Punkt recht und bezeichnet die mangelnde Auseinandersetzung mit den Strukturen des globalen Kapitalismus in den postkolonialen Ansätzen als „schwerwiegende Lücke“ (Hall 2002, S. 243-244). ${ }^{2}$

Die weite Definition ist jedoch nicht nur problematisch, weil sie ein leicht verzerrtes Bild von den tatsächlichen, meist auf diskursive Phänomene beschränkten Forschungen vermittelt. Sie vermag es auch nicht, die Postkolonialen Studien von jenen (i. d. R. marxistisch orientierten) Arbeiten, die mit den Konzepten des Neokolonialismus, des Imperialismus, der Dependenz oder des Weltsystems operieren, analytisch abzugrenzen. ${ }^{3}$ Angesichts der unterschiedlichen theoretischen (und oft auch wissenschaftstheoretischen) Ausrichtungen wäre eine solche Abgrenzung jedoch sinnvoll. Gewiss nicht alle, aber viele dieser Arbeiten beruhen auf einem „ökonomistischen, teleologischen und letztendlich reduktionistischen Marxismus“ (Hall 2002, S. 243), dessen Krise Hall zufolge eine wichtige Rolle in der Entstehung der Postkolonialen Studien gespielt hat. Wissenschaftstheoretisch stehen sie zumindest implizit in der Regel genau den positivistischen Grundannahmen (Objektivismus, Empirizismus, Naturalismus) nahe, die Postkoloniale Studien oft in Frage stellen, da sie die materielle Wirklichkeit als diskursiv vermittelt

2 Auf einige neuere Arbeiten der Postkolonialen Studien, die eine solche Auseinandersetzung suchen, wird in Abschnitt 5 eingegangen.

3 Interessanterweise sind in diesem Kontext Arbeiten aus dem globalen Süden - v. a. die lateinamerikanische Dependenztheorie - durchaus von kritischen AutorInnen der deutschen Politikwissenschaft der 1970er-Jahre rezipiert worden. 
begreifen. In ihren theoretischen Konzeptionen beinhalten sie des Öfteren Dichotomien, klassischerweise zwischen aktiven Kolonisierenden und passiven Kolonisierten, die zahlreiche postkoloniale Ansätze problematisieren.

Eine Definition des Forschungsfelds Postkolonialer Studien sollte also erstens die konstatierte Lücke anerkennen, zweitens sie zu schließen versuchen und drittens die Unterschiede zu (neo-)marxistischen Ansätzen deutlich machen. Demnach ließe sich formulieren: Postkoloniale Studien untersuchen die koloniale Prägung von Repräsentationen und Identitäten sowie ihr Verhältnis zu materiellen Praktiken auch nach der formellen Dekolonisierung unter Infragestellung von positivistischen Grundannahmen und theoretischen Dichotomien.

Auch wenn hier von einer Lücke hinsichtlich eines Großteils der postkolonialen Ansätze gesprochen wird, so muss doch in aller Deutlichkeit festgehalten werden, dass sie damit keinesfalls als defizitär oder nicht ernst zu nehmen gekennzeichnet werden sollen (wie es in einigen der radikalen Kritiken suggeriert wird). Die Arbeiten von Said, Spivak, Bhabha und zahlreichen anderen TheoretikerInnen haben sich ungeachtet ihrer weitgehenden Beschränkung auf die diskursive Ebene unschätzbare Verdienste erworben, indem sie mit ihren Konzepten wie Orientalismus, Subalternität und Hybridität auf schwerwiegende blinde Flecken, eurozentrische Annahmen und Verkürzungen in der Darstellung von nachkolonialen Gesellschaften und Nord-Süd-Beziehungen aufmerksam gemacht haben - gerade im Hinblick auf die Frage, aus welcher Perspektive Wissensproduktion stattfindet. Was an dieser Stelle behauptet wird, ist vielmehr, dass ihre Verknüpfung mit der empirischen Analyse materieller Praktiken bisher nur unzureichend stattgefunden hat, sich aber als in hohem Maße erkenntnisfördernd erweisen kann.

\section{Politikwissenschaft und postkoloniale Studien: komplementäre Defizite}

Ich vertrete die These, dass die Verknüpfung von Postkolonialen Studien und Politikwissenschaft vielversprechend ist, da in den allermeisten Arbeiten der beiden Bereiche Defizite zu finden sind, die in dem jeweils anderen nicht vorliegen, ihre Stärken und Schwächen daher komplementär sind. Während die Stärke politikwissenschaftlicher Arbeiten im Bereich der Nord-Süd-Beziehungen in der systematischen und gründlichen Durchdringung ihrer empirischen Gegenstandbereiche liegt, ist eine Schwäche in der mangelnden Sensibilität für die Relevanz des Kolonialismus und den Einfluss kolonialer Denkmuster auch lange nach der formalen Dekolonisierung auszumachen. Umgekehrt ist die theoretisch fundierte Untersuchung kolonialer Kontinuitäten die zentrale Stärke postkolonialer Arbeiten, die jedoch bei der Untersuchung politischer Institutionen und Prozesse oftmals unsystematisch und oberflächlich vorgehen. Selbstverständlich gibt es in beiden Bereichen Ausnahmen, auf die im nächsten Abschnitt einzugehen sein wird. Da diese Ausnahmen im Bereich der Postkolonialen Studien häufiger anzutreffen sind als in der Politikwissenschaft, ist die Diagnose eines komplementären Defizits leicht asymmetrisch: Defizite sind in beiden Bereichen vorhanden, erscheinen im zweiten jedoch gravierender als im ersten. Als grundlegende These wird diese Behauptung in diesem Abschnitt exemplarisch durch einige Arbeiten aus den Bereichen illustriert. Für die Politikwissenschaft untersuche ich daher einige Texte von Her- 
fried Münkler und Ulrich Menzel, für die Postkolonialen Studien von Nikita Dhawan, Cheryl McEwan und Teivo Teivainen. Es sei in aller Deutlichkeit darauf hingewiesen, dass die Kritikpunkte nicht allein die hier behandelten AutorInnen betreffen. Diese sind lediglich ausgewählt worden, um die Tendenzen der von ihnen hier repräsentierten wissenschaftlichen Bereiche an einigen ihrer prominenten und verdienstvollen VertreterInnen aufzuzeigen. ${ }^{4}$ Es geht daher an dieser Stelle nicht um Kritik an ihren vermeintlichen Versäumnissen, sondern um die Frage, welche Schwächen in der Politikwissenschaft und in den Postkolonialen Studien generell auffindbar sind, und wie ihre Stärken (die sich gerade auch in den Arbeiten der zitierten AutorInnen manifestieren) besser zur Geltung gebracht werden können.

Herfried Münkler ist sicher einer der bekanntesten deutschen Politikwissenschaftler und Professor für Theorie der Politik an der Humboldt-Universität zu Berlin. Sein Buch Die neuen Kriege von 2002 war nicht nur im Buchhandel ein Bestseller, sondern wird auch von der Bundeszentrale für politische Bildung vertrieben und kann als ein einflussreiches und weitverbreitetes Standardwerk in der deutschsprachigen Debatte zu Frieden und Konflikten gelten, das auch in vielen Lehrveranstaltungen Erwähnung findet. Grundlegend ist in diesem Buch die Gegenüberstellung vom „klassischen Staatenkrieg“ (S. 7) und den „neuen Kriegen“ (S. 9), die "gegen den klassischen Staatenkrieg abgegrenzt" werden müssen. Die „Besonderheiten der neuen Kriege“ sieht Münkler demnach in der „Entstaatlichung bzw. Privatisierung kriegerischer Gewalt“, der „Asymmetrisierung kriegerischer Gewalt“ und der „Autonomisierung vordem militärisch eingebundener Gewaltformen" (S. 10-11).

Im weiteren Verlauf der Argumentation wird deutlich, dass die Beschreibung der hier gegenübergestellten Gewaltformen nicht nur verschiedenen Epochen, sondern auch geografischen Regionen bzw. sogar Kulturen zugeordnet wird. Ursächlich verantwortlich für die neuen Kriege seien „der Mangel an integren und korruptionsresistenten Eliten“ (S. 16) und der „Tribalismus sozial wie kulturell unzureichend integrierter Gesellschaften" (S. 19), die zu gescheiterten Staatsbildungsprozessen geführt haben. Die Diagnose legt nahe, dass die Menschen in den entsprechenden Ländern anscheinend nicht in der Lage sind, sich auf eine verantwortungsvolle und demokratische Weise selbst zu regieren - was, dies sei bereits an dieser Stelle angemerkt, die zentrale Legitimation kolonialer Herrschaft war (vgl. Doty 1996).

Münkler beschreibt einen „Gegensatz“ zwischen einerseits den europäischen Kriegen der frühen Neuzeit und den außereuropäischen neuen Kriegen und andererseits "den Staatenkriegen, wie sie in Europa von der Mitte des 17. bis zum Anfang des 20. Jahrhunderts geführt wurden“" (S. 24) hinsichtlich ihrer Begrenztheit und v. a. Regelhaftigkeit. Gerade die hier etablierte Unterscheidung zwischen Soldaten und Zivilbevölkerung werde in den neuen Kriegen jedoch missachtet:

4 Es ließe sich einwenden, dass die ausgewählten AutorInnen der Postkolonialen Studien nicht ganz den gleichen Stellenwert haben wie Menzel und Münkler innerhalb der deutschen Politikwissenschaft. Allerdings erfahren die Arbeiten von McEwan international und Dhawan hierzulande zunehmende Beachtung und Verbreitung und gelten bereits oftmals als Standardwerke. 
Letztere würden „durch exzessive Gewalt eingeschüchtert“ (S. 29), zentrales Mittel des Krieges sei „nicht länger die Entscheidungsschlacht, sondern das Massaker“ (S. 29), an der Tagesordnung seien nicht nur Raub und Plünderung, sondern „beinahe alltäglich gewordene Vergewaltigungsorgien“, „Verstümmelungen der Opfer“ und die „Trophäisierung menschlicher Körperteile“ (S. 30).

Münkler unterscheidet nicht nur zwischen zwei verschiedenen Arten der Kriegsführung, die er einerseits mit Westeuropa und Nordamerika und andererseits mit der Peripherie assoziiert, sondern er beschreibt die eine Form als gekennzeichnet durch Regeln und Begrenzungen der Gewalt - man könnte sagen als ,zivilisiert $^{\varsigma}$ - und die andere als entgrenzt, irrational und unmenschlich - als ,barbarisch'. Auch wenn er die Begriffe nicht benutzt, seine Beschreibungen legen diese Bezeichnungen unweigerlich nahe. Die diese Zweiteilung widerlegenden Praktiken im Zweiten und Ersten Weltkrieg werden von Münkler zwar an einer Stelle beiläufig erwähnt, jedoch spricht er weiterhin von „unserer Vorstellung “ des Krieges (S. 24), wobei er sich offenbar auf eine westeuropäisch-nordamerikanische, westliche Kultur bezieht, da in den neuen Kriegen in der Peripherie die Gewalt laut Münkler in den Händen von Akteuren liegt, denen diese Vorstellungen fremd sind (S. 11).

Münklers Thesen zu den neuen Kriegen sind in der politikwissenschaftlichen Debatte zwar oft und durchaus fundiert kritisiert worden (vgl. Schlichte 2002, 2006; Chojnacki 2004; Kahl u. Teusch 2004; Daase 2003), aber erst aus einer postkolonialen Perspektive, die nach der Kontinuität kolonialer Denkmuster fragt, wird die gesamte Tragweite der argumentativen Verkürzungen und blinden Flecken deutlich: Mit keiner Silbe geht Münkler in diesem Kontext auf die verbrecherische Kriegsführung und die exzessive Gewalt gegen die Zivilbevölkerung bis hin zum Völkermord ein, die von Seiten der europäischen Staaten im Rahmen des Kolonialismus ausgeübt wurde (vgl. Moses u. Stone 2006; Plumelle-Uribe 2004). Stattdessen wird das Bild gezeichnet, dass die europäischen Staaten nach den grausamen Staatsbildungskriegen des 17. Jahrhunderts einen Zivilisierungsprozess durchgemacht haben, der in die Haager Landkriegsordnung und die Genfer Konvention mündete, während in anderen Ländern v. a. aufgrund ihrer defizitären politischen Kultur dieser Zivilisierungsprozess nicht stattfand bzw. scheiterte, weshalb dort heute eine ,barbarische“ Form der Kriegsführung anzutreffen ist, die mit der ,zivilisierten' Kriegsführung Europas nichts mehr gemein hat. Die Ausblendung der Massaker im Kolonialismus ermöglicht die Gegenüberstellung einer ,zivilisierten` europäischen Kriegsführung und einer , unzivilisierten' nichteuropäischen. Hier wird somit auf Konstruktionen und Argumentationsmuster der kolonialen Ära (das zivilisierte Selbst vs. das barbarische Andere) zurückgegriffen. Eine Auseinandersetzung mit den Postkolonialen Studien hätte in diesem Kontext eine Vermeidung dieser Muster und somit eine Fokussierung der Analyse auf die tatsächlich relevanten Erklärungsfaktoren ermöglicht.

Das zweite Beispiel betrifft den Bereich der Entwicklungstheorie und Entwicklungspolitik, und hier die Schriften von Ulrich Menzel, Professor für Internationale Beziehungen an der TU Braunschweig und neben Franz Nuscheler der wohl profilierteste Politikwissenschaftler in diesem Politikfeld. Seine auch heute noch als Referenz dienenden und vielzitierten Arbeiten aus den 1990er-Jahren (1992, 
1993) sowie sein kürzlich erschienener Beitrag in einem Lehrbuch (2010) können hier als typisch für eine breite Strömung politikwissenschaftlicher Entwicklungstheorie gelten. Auch hier sind, so die hier vertretene These, Argumentationsmuster zu finden, die eine unverkennbare Nähe zu aus der Ära des Kolonialismus bekannten gedanklichen Konstruktionen aufweisen.

Menzel definiert die Unterdisziplin der Entwicklungstheorie wie folgt:

„Ich verstehe unter Entwicklungstheorie Aussagen, mit deren Hilfe [...] begründet wird, warum es in den Industriegesellschaften Westeuropas, Nordamerikas und Ostasiens zu Wirtschaftswachstum, Industrialisierung, sozialer Differenzierung und Mobilisierung, mentalem Wandel, Demokratisierung und Umverteilung gekommen ist (diese Prozesse nennt man Entwicklung) bzw. warum in übrigen Teilen der Welt diese Prozesse ausbleiben, nur unvollständig realisiert werden oder lediglich eine Karikatur dieser Prozesse zu beobachten ist.“ (Menzel 1993, S. 132).5

Wir haben es in diesem Zitat einerseits mit einem gedanklichen Konstrukt, andererseits mit einer normativen Setzung zu tun. Das gedankliche Konstrukt identifiziert einen universellen Prozess sozialen Wandels nach dem Vorbild Westeuropas und Nordamerikas und somit eine universelle Entwicklungsskala, an deren Spitze die industrialisierten Gesellschaften stehen. Dies ist zutreffend als das "Colonizer's model of the world“ (Blaut 1993) und von zahlreichen Arbeiten aus dem Bereich der Postkolonialen Studien (z. B. Chakrabarty 2000) und des PostDevelopment (z. B. Sachs 1993) als eurozentrisch kritisiert worden. Das Konstrukt negiert die Kontingenz und Heterogenität historischer Prozesse sozialen Wandels. In ihm finden sich Elemente des evolutionistischen Denkens der europäischen Sozialwissenschaften v. a. des 19. Jahrhunderts: Die Anderen sind so, wie wir früher waren, und sie werden (wenn sie sich dem Fortschritt öffnen) später so werden wie wir jetzt sind - dies manifestiert sich bei Hegel, Marx, Spencer und Comte in ganz ähnlicher Weise (vgl. Ziai 2004). Nandy bezeichnet dies als eine „transformation of geocultural differences into historical stages“ (1992, S. 146), Melber spricht hier von einer "Verzeitlichung des räumlichen Nebeneinander" (1992, S. 20). Menzels Darstellung sozialen Wandels ist von genau dieser eurozentrischen Sichtweise geprägt.

Dieser Befund wird verstärkt, wenn wir die normative Dimension einbeziehen. Der Autor geht (wie auch nahezu die gesamte Disziplin der Entwicklungstheorie) von einer positiven normativen Konnotation von „Entwicklung“ aus. Die ,weniger entwickelten' Gesellschaften erscheinen so nicht nur als historisch rückständig, sondern als defizitär, als minderwertige Abweichung von der idealen Norm, die die eigene Gesellschaft darstellt. Damit ist aber die ,gute Gesellschaft' kurzerhand mit der ,westlichen' bzw. mit der industrialisierten, kapitalistischen, modernen, demokratischen und sozialstaatlich abgesicherten Massenkonsumgesellschaft ineinsgesetzt - ohne zu berücksichtigen, dass es möglicherweise andere Vorstellungen einer guten, lebenswerten Gesellschaft geben könnte. Oligarchischer Konsum und die entmündigenden Aspekte der Formulierung universeller gesellschaft-

5 Auch in neueren Arbeiten (v. a. Menzel 2010, S. 11-16 und passim) finden sich sehr ähnliche Begriffsgrundlegungen. 
licher Zielvorstellungen (das ,Sprechen für Andere`) bleiben ganz außen vor. Diese normative Setzung der eigenen Gesellschaft als positive Norm, gegenüber der die defizitären Gesellschaften des Südens (durch ,Entwicklungspolitik') angeglichen werden müssen, verdeutlicht, dass das Projekt der ,Entwicklung der Unterentwickelten' eine modifizierte Weiterführung der Mission der ,Zivilisierung der Unzivilisierten' war, die sich nach dem Holocaust und der Allgemeinen Erklärung der Menschenrechte jedoch vom kolonialen Rassismus und der damit verbundenen Behauptung der Unfähigkeit bestimmter Völker, sich selbst zu regieren, zunehmend gelöst hat.

Allerdings wird diese Ablösung auch in der Entwicklungspolitik bisweilen wieder infrage gestellt, u. a. in den Schriften Menzels. In seinen „Vorschlägen zu einer grundlegenden Neuorientierung der Nord-Süd Politik“ (1992, S. 202-213) spricht er sich für „Interventionen in den Krisengebieten der Welt von seiten der führenden Industrieländer des Westens“ aus (S. 209). Dabei sollen „besonders bedrohte Krisenregionen [...] bis auf weiteres der Treuhandschaft der Länder des Nordens “ unterstellt werden (S. 211), verbunden mit einer entsprechenden „partiellen und zeitweisen Einschränkung der Souveränität der einheimischen Behörden “ (S. 211). Die demokratische Legitimation von entsprechenden Interventionen und Treuhandschaftsmodellen soll explizit nicht durch die UN, sondern durch „die hiesigen Parlamente“ (des Nordens) geschehen (S. 211). Den Regierungen des Nordens wird implizit der Wille und die Fähigkeit zur selbstlosen Verwirklichung des Allgemeinwohls zugesprochen, während den Regierungen des Südens diese Eigenschaften ebenso pauschal aberkannt werden. Begründet wird dies nur mit Hinweisen auf die „korrupten Eliten“ (S. 205) bzw. „despotischen [...] Traditionen” (S. 220) im Süden. ${ }^{6}$ Dies ist jedoch ein historisch allzu bekanntes Argumentationsmuster.

Auch hier hätte ein Mindestmaß an Sensibilität für postkoloniale (oder PostDevelopment-) Ansätze dazu geführt, dass sich die Diskussion auf die in dem Beitrag angesprochenen tatsächlich relevanten politischen und politikwissenschaftlichen Fragen des Verhältnisses von Menschenrechten und Souveränität, einer „Responsibility to protect“ der internationalen Gemeinschaft oder einer grundlegenden Transformation (oder gar Abschaffung) der Entwicklungszusammenarbeit fokussiert, anstatt implizit koloniale Stereotypen zu reproduzieren. Leider vergibt Menzel diese Möglichkeit durch die Abwertung dieser und ähnlicher Ansätze zu „Modethemen“ (S. 1992, S. 130-131, 2010 S. 148).

Die in beiden hier behandelten Beispielen sichtbare mangelnde Sensibilität gegenüber postkolonialen Fragestellungen und Konzepten ist in der Politikwissenschaft verbreitet (auf die Ausnahmen komme ich noch zu sprechen). Andererseits ist in den Postkolonialen Studien ein komplementäres Defizit festzustellen: Diejenigen unter ihnen, die sich überhaupt mit im engeren Sinne empirischen Gegenstandsbereichen der Politikwissenschaft beschäftigen, weisen oftmals eine unsystematische oder oberflächliche Auseinandersetzung mit politischen Institutionen

6 Auch hier finden sich in neueren Schriften ähnliche Argumentationslinien (Menzel 2010, S. 154155). 
und Prozessen auf. Auch diese These möchte ich im Folgenden an einigen Beispielen illustrieren.

Nikita Dhawan kann als eine bekanntesten VertreterInnen der Postkolonialen Studien im deutschsprachigen Raum gelten. Sie ist Professorin für Gender und Postkoloniale Studien an der Goethe-Universität Frankfurt und hat (zusammen mit Maria do Mar Castro Varela) das erste deutschsprachige Einführungsbuch in die Postkolonialen Theorien verfasst (Castro Varela u. Dhawan 2005), in dem die verschiedenen theoretischen Ansätze wie auch die gegenüber ihnen geäußerten Kritikpunkte ebenso kompetent wie verständlich vorgestellt werden. Für eine Untersuchung der Postkolonialen Studien hierzulande erscheint sie somit kaum verzichtbar. In dem ebenfalls von den beiden Autorinnen herausgegebenen Schwerpunktheft der femina politica zu Feministischer Postkolonialer Theorie ist auch Dhawan mit einem Beitrag zu „Dekolonisierung und Demokratisierung“ vertreten, in dem sie „eine postkolonial-feministische Analyseperspektive“ einnimmt, „um die Machtverhältnisse aufzuzeigen, die gegenwärtige Diskurse von globaler Entwicklungszusammenarbeit begleiten. Der Fokus soll hierbei auf der Frage liegen, inwiefern Entwicklungsarbeitende und die internationale Zivilgesellschaft in hegemoniale Diskurse verstrickt bleiben, während sie vorgeben, für marginale Gruppen einzutreten“ (Dhawan 2009, S. 54). Diese Fragestellung wird dann in dem Beitrag über eine Auseinandersetzung mit Martha Nussbaum, Ulrich Beck, Nancy Fraser und Sheila Benhabib verfolgt, v. a. aber unter Berufung auf die postkoloniale Theoretikerin Gayatri Spivak, die insgesamt 33-mal zitiert wird und deren Schriften mehr als ein Drittel des Literaturverzeichnisses ausmachen.

Dhawans Argumentation ist an einigen Stellen durchaus schlüssig und erhellend, aber der zentrale Punkt ist, dass ihre Analyse des Entwicklungsdiskurses weder auf die einschlägige sozialwissenschaftliche Literatur zum Thema eingeht (z. B. Moore u. Schmitz 1995; Crush 1995; Escobar 1995; Cowen u. Shenton 1996; Cooper u. Packard 1997; Mosse u. Lewis 2005; Ziai 2006) noch in irgendeiner Form empirisch auf Dokumente der Entwicklungszusammenarbeit eingeht. Die Auseinandersetzung mit dieser Literatur und v. a. den entsprechenden Primärquellen würde ihre - oftmals durchaus nicht unberechtigte - Kritik deutlich fundierter und präziser werden lassen. So wartet die Leserin nach der These „Die ,Politik des Helfens' verdeckt ökonomische und geopolitische Interessen, wobei ,Geschlecht und Entwicklung' dem globalen Norden als Alibi dient, im globalen Süden erneut zu intervenieren“ (Dhawan 2009, S. 55) vergeblich auf die empirischen Belege.

Auch ihre Auseinandersetzung mit Demokratisierungsprozessen in Ländern des Südens beruht nur auf einer schmalen empirischen Basis - sie zitiert einen Artikel über die nicaraguanischen Wahlen 1990. Wiederum ist die vertretene These, dass nämlich „der Wahlprozess im globalen Süden durch die Vernachlässigung der Frage neokolonialer Abhängigkeit die Subalternität in jenem Moment reproduziert, in welchem den Subalternen scheinbar ermöglicht wird, zu sprechen “ (Dhawan 2009, S. 57) durchaus interessant und im Hinblick auf ihr Beispiel nicht unplausibel. Aber wiederum versäumt es die Autorin, durch die Berücksichtigung der politikwissenschaftlichen Literatur und der entsprechenden empirischen Beispiele (z. B. Gills et al. 1993; Hippler 1994; Schubert u. Tetzlaff 1998; Merkel 
1999; Abrahamsen 2000) ihre These zu untermauern und in der wissenschaftlichen Debatte zu verorten.

Durch die wenig ausgeprägte empirische Fundierung ihrer Argumentation bestätigt Dhawan in diesem Text den altbekannten Vorwurf gegenüber den Postkolonialen Studien, sich nicht hinreichend mit materiellen Strukturen auseinanderzusetzen. Den folgenden beiden Texten ist dieser Vorwurf auf den ersten Blick kaum zu machen.

Cheryl McEwan hat sich ebenfalls als Autorin eines Einführungsbuchs einen Namen gemacht und hat mit Postcolonialism and Development (2009) einen größtenteils überaus gelungenen - Versuch vorgelegt, das Feld der Entwicklungspolitik aus einer postkolonialen Perspektive zu betrachten. Die große Beachtung, die das Buch in der wissenschaftlichen Debatte gefunden hat, die vielen positiven Rezensionen und seine zunehmende Verwendung als Lehrbuch rechtfertigen seine eingehendere Untersuchung an dieser Stelle. McEwan befasst sich in ihm an zahlreichen Stellen mit den Institutionen und Prozessen der Entwicklungspolitik und könnte daher potenziell die hier vertretene These einer mangelhaften Auseinandersetzung postkolonialer Arbeiten mit politikwissenschaftlichen Gegenstandsbereichen widerlegen.

Dies ist jedoch nur zum Teil der Fall, denn die Behandlung der politischen Institutionen und Prozesse geschieht nicht immer auf eine gänzlich souveräne und überzeugende Art und Weise. Zum Teil sind gewisse Ungenauigkeiten in dieser Auseinandersetzung festzustellen: Die WTO gehört nicht wie behauptet zu den Bretton-Woods-Institutionen (McEwan 2009, S. 129), da die auf der BrettonWoods-Konferenz 1944 anvisierte International Trade Organisation am Veto des US-Congress scheiterte - die WTO wurde erst 1995 als Resultat der UruguayRunde des General Agreement on Tariffs and Trade (GATT) gegründet. Die Millennium Development Goals gehen keinesfalls auf die Weltbank zurück (S. 168), sondern auf ein Strategiepaper des Development Aid Committee der OECD von 1996. Die Proteste gegen die WTO in Seattle fanden nicht 2000 (S. 196), sondern bereits ein Jahr früher statt. Das Weltsozialforum findet nicht ausschließlich in Porto Alegre statt (S. 196), sondern fand z. B. 2004 in Mumbai, 2007 in Nairobi und 2009 in Belém statt und war 2006 und 2008 dezentral organisiert. Der $\mathrm{Hu}$ man Development Index des United Nations Development Program (UNDP) misst Kaufkraft (nicht Einkommen), Schulbildung, Alphabetisierung und Lebenserwartung, aber nicht die menschliche Freiheit (S. 91). Diese Ungenauigkeiten mögen im Einzelfall nicht allzu bedeutsam erscheinen, in ihrer Summe belegen sie jedoch einen Mangel an Sorgfalt in der Auseinandersetzung mit politischen Institutionen und Prozessen, der m. E. symptomatisch für einige postkoloniale Studien ist.

Zum anderen wirft auch McEwans Argumentation im Hinblick auf diese politischen Prozesse und Institutionen bisweilen Fragen auf: Wenn die „Anti-Globalisierungsproteste“ mit der postkolonialen Kritik an „Entwicklung“ in einen Topf geworfen werden (S. 196), so werden beide recht pauschal behandelt, v. a. in der (m. E. verfehlten, siehe Ziai 2010) Gleichsetzung von Neoliberalismus und „Entwicklung“. Wenn Weltbank, IWF und WTO als „Treuhänder des modernen Zeitalters" beschrieben werden, die die Verteilung von Ressourcen im Süden kontrol- 
lieren (S. 81), dann unterschlägt dies die zentrale Rolle nationaler Eliten, die mit der Dekolonisierung das Mandat der Treuhandschaft übernommen haben und oftmals viel direkter an der Ausübung von Gewalt im Namen von „Entwicklung“ (auf die McEwan auch aufmerksam macht, S. 193) beteiligt waren. ${ }^{7}$ Wenn unterstellt wird, Entwicklungsinstitutionen würden sich nicht dafür interessieren, wie die Armen selbst die Armut sehen (S. 92), dann ist es nicht ganz redlich, an dieser Stelle die diesbezügliche Befragung von 60.000 ,Armen' durch die Weltbank und die darauf aufbauende Studie „Voices of the Poor“ unerwähnt zu lassen. ${ }^{8}$ Auch wenn McEwans Arbeit zurecht als Meilenstein für die Darstellung und Verbreitung postkolonialer Sichtweisen in der Entwicklungsforschung gelten kann, führen die hier angeführten Kritikpunkte doch wieder zu einer Untermauerung der These des politikwissenschaftlichen Defizits vieler postkolonialer Studien.

Als drittes und letztes Beispiel soll eine Studie von Teivo Teivainen dienen, die in dem kürzlich erschienenen Sammelband Vom Imperialismus zum Empire (Randeria u. Eckert 2009) erschienen ist. Der Band verknüpft postkoloniale Fragestellungen mit Analysen von Globalisierungsprozessen und erschließt (als Suhrkamp-Taschenbuch) breite Leserschichten für die Thematik. Teivainens Studie in diesem Band untersucht die Rolle des Internationalen Währungsfonds (IWF) in den Nord-Süd-Beziehungen und befasst sich somit explizit mit einem politikwissenschaftlichen Thema, weshalb er an dieser Stelle nähere Betrachtung erfährt. Auch hier sind Teile der Argumentation durchaus spannend und überzeugend: Teivainen argumentiert, dass einerseits die „Doktrin der ökonomischen Neutralität" (Teivainen 2009, S. 107), die wirtschaftspolitische Auflagen als unpolitische Expertenratschläge konzipiert, und andererseits die Vorstellung vom Nationalstaat als der maßgeblichen politischen Ebene (und entsprechend der internationalen Politik als einer Ebene, in der andere Maßstäbe gelten) die Aufrechterhaltung des undemokratischen, nach Kapitalanteilen gewichteten Stimmrechtsystems des IWF ermöglicht. Sein wichtigstes Argument jedoch betrifft die Rolle des IWF in einer globalisierten Welt, in der Machtstrukturen zunehmend auf die internationale Ebene verlagert werden, Mitbestimmungsmöglichkeiten jedoch nationalstaatlich begrenzt bleiben: „Diese Globalisierung der Machtstrukturen schafft, so meine These, ein globales System der Governance mit einer Anzahl von hierarchischen und autoritären Entscheidungsprozessen, die über staatliche Grenzen hinausgreifen" (Teivainen 2009, S. 128-129). Der IWF sei in diesem Kontext aufgrund der neoliberalen Ausrichtung seiner Konditionalität ein Instrument der „Normierung und Disziplinierung der kreditnehmenden Regierungen“ (S. 132): „Die Beziehung zwischen dem IWF und einer kreditnehmenden Regierung eines Landes der Dritten Welt ist nicht die Begegnung zweier Subjekte, die auf einem privaten Markt frei agieren können, sondern eher eine politische Knechtschaft, in

7 Hier wird auch die mangelnde staats- und akteurstheoretische Fundierung der Darstellung sichtbar.

8 Siehe http://web.worldbank.org/WBSITE/EXTERNAL/TOPICS/EXTPOVERTY/0,,contentMDK :20613045 isCURL:Y menuPK:336998 pagePK:148956 piPK:216618 theSitePK:336992,00. html 
der die kreditnehmende Regierung nur sehr begrenzte Handlungsfreiheit hat" (S. 132).

Mithin wird hier die These einer neokolonialen Weltordnung oder zumindest kolonialer Kontinuitäten im Hinblick auf die Rolle des IWF vertreten. Aber auch hier sind die empirischen Belege, trotz eines interessanten Vergleichs der Situation lateinamerikanischer Schuldnerstaaten in den 1920er- und 1930er-Jahren mit ihrer Situation im Rahmen der Schuldenkrise seit 1982, nicht gänzlich überzeugend, und zwar aus drei Gründen: Erstens wird nicht darauf eingegangen, dass gerade im Hinblick auf das Verhältnis der großen lateinamerikanischen Schwellenländer zum IWF weitreichende Veränderungen seit den 1980er-Jahren zu konstatieren sind. Argentinien und Brasilien z. B. haben ihre Kredite vorzeitig zurückgezahlt und unterliegen seit einigen Jahren keinerlei Auflagen des IWF.

Zweitens versäumt es die Analyse, detaillierter auf die Handlungsspielräume der kreditnehmenden Regierungen einzugehen, indem von ihrer Machtlosigkeit und einer vollständigen Erfüllung der IWF-Auflagen ausgegangen wird. Empirische Studien oder der Rückgriff auf die Fachliteratur wären hier hilfreich gewesen. So hat einerseits George (1988) die massiven Drohungen seitens der Geberländer gegenüber Regierungen aufgezeigt, die eine unilaterale Begrenzung des Schuldendienstes erwogen haben. Andererseits haben Mosley et al. (1991) in einer umfassenden Studie aufgezeigt, dass zumindest im Hinblick auf Kredite der Weltbank Nehmerregierungen oftmals Auflagen nur teilweise oder gar nicht erfüllt haben, ohne negative Konsequenzen zu erleiden. Hier jedoch bleibt es beim altbekannten Bild des dominanten IWF, das stärker belegt werden müsste.

Drittens bleiben die Transformations- oder institutionellen Lernprozesse innerhalb des IWF unberücksichtigt. Hier wäre zum einen auf die Reform der Strukturanpassungsprogramme im Rahmen der neuen Poverty Reduction and Growth Facility und ihre formelle (!) Ausrichtung auf Armutsbekämpfung, Partizipation und "ownership“ einzugehen, andererseits aber auf den Vorschlag der damaligen IWF-Vizepräsidentin Anne Krueger eines Sovereign Debt Restructuring Mechanism (SDRM), eines Staateninsolvenzverfahrens. Ein solches Verfahren ist seit langem von KritikerInnen des IWF gefordert worden, und auch wenn der SDRMVorschlag eine sehr viel weniger weitreichende Umstrukturierung des herrschenden Schuldenregimes und der damit verbundenen Machtverhältnisse bedeutet hätte als das von NGOs vorgeschlagene Schiedsverfahren FTAP (Fair and Transparent Arbitration Procedure), so passt diese Initiative nicht ohne Weiteres in das Bild des neokolonialen IWF. Interessant ist jedoch, dass der SDRM-Vorschlag im Exekutivdirektorium nicht nur am Veto der USA, sondern auch der lateinamerikanischen Schwellenländer selbst (denen ein solches Verfahren zugutekommen sollte) scheiterte. Sie befürchteten nämlich durch dessen Einführung eine negative Reaktion der internationalen Finanzmärkte in Form eines verschärften Risikoaufschlags auf die ihnen abverlangten Zinssätze. Offenbar ist die internationale politische Ökonomie zwar durchaus von asymmetrischen Machtverhältnissen gekennzeichnet, diese sind jedoch komplexer als das Bild der politischen ,Knechtschaft" der Schuldnerstaaten gegenüber dem IWF.

Dementsprechend kann auch in diesem Fall konstatiert werden, dass die aus einer postkolonialen Perspektive vorgenommene Analyse politischer Institutionen 
und Prozesse durch stärkeren Bezug auf die politikwissenschaftliche Literatur und v. a. auf systematische empirische Studien ihren Gegenstandsbereich sehr viel genauer hätte erfassen können.

Die These komplementärer Defizite in der Politikwissenschaft (mangelnde Sensibilität für postkoloniale Konzepte und Fragestellungen) und den Postkolonialen Studien (unsystematische und oberflächliche Auseinandersetzung mit politischen Institutionen und Prozessen) lässt sich durch die hier angeführten Beispiele bestätigen.

\section{Postkoloniale Politikwissenschaft: Stand der Forschung}

Ebenso wie die Postkolonialen Studien sich oft nur unzureichend mit politischen Institutionen und Prozessen auseinandergesetzt haben, hat sich die Politikwissenschaft kaum um eine Berücksichtigung der postkolonialen Ansätze bemüht - obwohl die Gegenstandsbereiche der nachkolonialen Gesellschaften und Nord-SüdBeziehungen durchaus als zu ihren Teildisziplinen zugehörig reklamiert werden. Hier müssen wir allerdings differenzieren: Die angelsächsische Political Science hat diesen Anspruch nicht, und die entsprechenden Disziplinen der International Relations und der Development Studies werden nicht als Teil von ihr angesehen, während im deutschsprachigen Raum Internationale Politik und Entwicklungspolitik durchaus Unterabteilungen der Politikwissenschaft sind. In beiden Fällen wird jedoch nicht nur die disziplinäre Abtrennung politischer und ökonomischer Phänomene deutlich (aufgehoben nur im Teilbereich Politische Ökonomie), sondern auch die (durch die konstitutive Rolle des Nationalstaats bedingte) Abtrennung der nationalen von der internationalen Sphäre und die in unserem Kontext besonders bedeutsame Orientierung an westlichen bzw. westeuropäisch-nordamerikanischen Gesellschaften als ,normaler' Gegenstand der Sozialwissenschaften. Die ,anderen' Gesellschaften werden von Spezialdisziplinen behandelt.

Im angelsächsischen Raum besteht eine Schnittmenge im Gegenstandsbereich also zwischen Postcolonial Studies einerseits und International Relations sowie Development Studies andererseits. Was den diesbezüglichen Stand der Forschung angeht, haben Darby und Paolini 1994 für die International Relations festgestellt: "both international relations and postcolonialism have been self-referential to an unusual degree [...] international relations and postcolonialism pass like ships in the night" (1994, S. 372, 384). Zwar skizzieren sie hypothetisch die Möglichkeit eines Brückenschlags durch die sog. Dritte Debatte in den Internationalen Beziehungen (vgl. Lapid 1989) und das mit ihr verbundene Interesse an Themen wie Kultur, Identität und Repräsentation (Darby u. Paolini 1994, S. 378), räumen aber andererseits ein, dass die erstaunliche Nichtbeachtung des Werks von Said (der bereits in den 1980ern breit rezipiert wurde) in der Disziplin wohl nicht nur auf disziplinäre Schranken, sondern auch auf grundlegende theoretische Differenzen zwischen seinem und den damals dominanten neorealistischen und -institutionalistischen Ansätzen v. a. hinsichtlich der Konzeptualisierung von Macht zurückzuführen ist (S. 381). Macht wird dort als materielle Ressource von Staaten begriffen und Repräsentation lediglich als neutrale Darstellung einer objektiven Realität. Obwohl ihrer Ansicht nach „a dialogue between the two discourses 
would be mutually provoking and therefore enriching “ (S. 372) - anscheinend naheliegend in den Bereichen Imperialismus, Orientalismus und Kultur (S. 378) und vielversprechend hinsichtlich der Fragen nach Macht und Repräsentation, Modernität und disziplinärer Politik (S. 384) - findet er schlichtweg nicht statt. Auch in seiner Analyse im Jahre 2000 kommt Darby zu dem gleichen ernüchternden Schluss: "postcolonialism [...] had practically no engagement with [the discipline of] international relations" (2000, S. 12).

Etwa zum gleichen Zeitraum untersucht Sylvester die Beziehungen zwischen Postcolonial Studies und Development Studies und konstatiert gleichermaßen, man habe es mit „two giant islands of analysis“ (1999, S. 703) zu tun, die völlig isoliert voneinander existierten, bzw. die sich sogar durch gänzlich verschiedene, komplementäre Sichtweisen auszeichneten: "one field begins where the other refuses to look" (S. 704). Salopp bringt sie diese Verschiedenheit folgendermaßen auf den Punkt: "development studies does not tend to listen to subalterns and Postcolonial Studies does not tend to concern itself whether the subaltern is eating" (S. 703) - die eine Disziplin beschränke sich auf Verbesserungen des Lebensstandards der Marginalisierten und bleibe dabei in eurozentrischen und expertengläubigen Konstrukten gefangen, während die andere sich ausgiebig mit der Kritik dieser Konstrukte, aber nicht mit den drängenden Fragen materiellen Elends befasse. Das mag etwas überspitzt erscheinen, ist von der Tendenz her jedoch durchaus zutreffend. Auf der theoretischen Ebene führt Sylvester die Differenzen v. a. auf den Einfluss des poststrukturalistischen Denkens in postkolonialen Ansätzen zurück und die damit verbundene Skepsis gegenüber dem zumindest dem größten Teil der Development Studies inhärenten Universalismus (S. 714). Dem gegenüber plädiert sie für eine Integration der Anliegen der Development Studies in die Agenda der Postcolonial Studies, denn „Postcolonial Studies, having neglected direct attention to such issues and their possible solutions - even in an imaginative or theoretical way - is nonetheless better placed than any Western agency to reinvent or recover postcolonial agendas of material well-being that matter on the ground" (S. 718).

Rund ein Jahrzehnt später entsprechen die apodiktischen Aussagen von Darby und Sylvester zum Verhältnis von Postkolonialen Studien und International Relations bzw. Development Studies zumindest im angelsächsischen Sprachraum jedoch nicht mehr ganz dem Stand der Forschung. Auch wenn ihre Thesen keineswegs völlig überholt sind, muss doch anerkannt werden, dass in den letzten Jahren einige Forschungsarbeiten publiziert wurden, die die Ansätze miteinander zu verknüpfen versucht haben.

Für die International Relations sind hier besonders die Sammelbände von Chowdhry und Nair (2004) und Gruffydd Jones (2006a) sowie die Arbeiten von Razack (2004), Biswas (2001), Grovogui (2007), Ling (2002), Agathangelou (2006) und Agathangelou und Ling $(2004,2009)$ zu nennen. In ihnen werden postkoloniale Fragestellungen und Konzepte produktiv auf Gegenstandsbereiche der International Relations angewandt wie z. B. den Vertrag zur Nichtverbreitung von Atomwaffen (Biswas 2001), die Asiatische Finanzkrise (Ling 2004), die Debatte um Sozialstandards und Kinderarbeit im Welthandel (Chowdhry 2004), Menschenrechte in Burma (Nair 2004), den Konflikt um den Suez-Kanal (Grovogui 
2007), ökonomische Globalisierung und Migration im Mittelmeerraum (Agathangelou 2004, 2006), den Irakkrieg (Gathii 2006), die UN-Intervention in Somalia (Razack 2004) und den Krieg gegen den Terror nach dem 11. September 2001 (Agathangelou u. Ling 2004). Die Arbeiten können an dieser Stelle nicht im Einzelnen dargestellt und diskutiert werden, Folgendes lässt sich jedoch ganz allgemein festhalten: Die empirische Forschung auf der Grundlage postkolonialer Ansätze findet hier in unterschiedlich systematischer Weise statt, und auch die analytische Abgrenzung zu antikolonialen Ansätzen ist bisweilen wenig ausgeprägt (exemplarisch: Gruffydd Jones 2006b), aber die Arbeiten demonstrieren eindrucksvoll die Möglichkeit und den Erkenntnisgewinn einer solchen Perspektive.

Für den Bereich der Development Studies ist zunächst zu konstatieren, dass der Einfluss postkolonialer Perspektiven sich primär in der Verbreitung und Berücksichtigung der sog. Post-Development-Ansätze manifestiert, die - obwohl weitestgehend isoliert von anderen postkolonialen Theorien (Sidaway 2007) - inhaltlich als postkoloniale Kritik der Entwicklungspolitik angesehen werden können. Als bekannteste VertreterInnen dieses Ansatzes können Esteva (1987), Escobar (1995), Rahnema (1997) und Sachs (1993) gelten, auch Mies und Shiva (1993) können dazugezählt werden (zur Debatte siehe Ziai 2004 und 2007). Allerdings ist dazu zu sagen, dass die Post-Development-Ansätze z. T. eher als intuitive und vereinfachte Anwendung postkolonialer Konzepte gelten können. Geeignetere Beispiele für Anwendungen postkolonialer Theorie finden sich bei White (2002), Goudge (2003), Eriksson Baaz (2005), Kothari (2005, 2006a, 2006b), Murray Li (2007), Kapoor (2008) und McEwan (2009). In ihnen geht es u. a. um rassistische Praktiken von EntwicklungshelferInnen (Goudge 2003), Rekrutierungspraktiken von Entwicklungsorganisationen (Kothari 2005), Projekte nachhaltiger Entwicklung in Indonesien (Murray Li 2007), Partizipation in der Entwicklungszusammenarbeit (Kapoor 2008) oder die Aktivitäten der Weltbank (McEwan 2009). Auch hier wird die Produktivität einer (impliziten oder expliziten) postkolonialen Perspektive aufgezeigt, doch auch hier sind bisweilen einige der bereits erwähnten Unzulänglichkeiten auffindbar: bei den Post-Development Ansätzen bisweilen Rückfälle in dichotome Argumentationsmuster, bei den anderen bisweilen eine Beschränkung auf die Ebene der Repräsentationen und Identitäten (Eriksson Baaz 2005) oder eine unvollkommene Vertrautheit mit den Strukturen der internationalen politischen Ökonomie (McEwan 2009). Auch hier ist demnach zu konstatieren, dass eine systematische Erforschung des Gegenstandsbereichs mittels postkolonialer Konzepte und Fragestellungen alles andere als obsolet ist.

Wenn wir uns nun dem deutschsprachigen Raum zuwenden, müssen wir feststellen, dass die Rezeption postkolonialer Ansätze hierzulande bei Weitem nicht den Stand der angelsächsischen Diskussion erreicht. Die entsprechenden Arbeiten sind fast ausschließlich innerhalb der Literatur-, Geschichts- und Kulturwissenschaften wahrgenommen worden. Die wenigen nicht-literaturwissenschaftlichen deutschsprachigen Monografien und Sammelbände (Conrad u. Randeria 2002; Steyerl u. Gutierrez Rodriguez 2003; Castro Varela u. Dawahn 2005; Ha et al. 2007; Golly u. Cohrs 2008; Randeria u. Eckert 2009) belegen dies. In der Soziologie gibt es erste Anzeichen einer Öffnung für postkoloniale Ansätze (siehe v. a. Costa 2005; Reuter u. Wieser 2006; Reuter u. Villa 2009; sowie die Beiträge von 
Randeria), Ähnliches gilt für die Geografie (vgl. z. B. Lossau 2002), in der Politikwissenschaft hingegen sind postkoloniale Ansätze bisher weitestgehend marginalisiert. ${ }^{9}$ Zwar wurde kürzlich die erste Juniorprofessur für Gender und Postcolonial Studies an einem politikwissenschaftlichen Institut in Deutschland eingerichtet, bezeichnenderweise aber mit einer Philosophin, Nikita Dhawan, besetzt. Von ihr ging auch die im Juni 2009 erfolgte Gründung des „Frankfurt Research Center for Postcolonial Studies" aus. Als weitere Ausnahmen sind die PolitikwissenschaftlerInnen Kien Nghi Ha mit seinen Arbeiten zur Migrationspolitik aus postkolonialer Perspektive (Ha 1999, 2004), Ina Kerner mit ihren Studien zu Feminismus und Entwicklungszusammenarbeit sowie Intersektionalität (Kerner 1999, 2009) sowie (in Österreich) Petra Purkarthofer mit ihrer Forderung der Verknüpfung rassismuskritischer, feministischer und materialistischer Ansätze in der Internationalen Politischen Ökonomie (Purkarthofer 2009) zu nennen, ebenso Jörg Meyer (2008) und Britta Krause in der Konfliktforschung.

Auch im Hinblick auf die deutschsprachigen Veröffentlichungen ist festzustellen, dass eine Vielzahl von interessanten Beiträgen und Einsichten geliefert wird, eine systematische empirische Forschung zu politischen Strukturen aus postkolonialer Perspektive jedoch noch weitestgehend aussteht. Politikwissenschaft und Postkoloniale Studien sind im deutschen Sprachraum noch weitestgehend voneinander isoliert.

\section{Anwendung postkolonialer Konzepte und Fragestellungen auf empirische Bereiche der Politikwissenschaft}

Anknüpfend an den hier dargestellten Stand der Forschung erscheint es sinnvoll, die Ansätze der Postkolonialen Studien mit der empirischen Erforschung klassischer Gegenstandsbereiche der Politikwissenschaft (v. a. der Internationalen Politik und der Entwicklungspolitik) zu verknüpfen. Zentraler Mechanismus dieser Verknüpfung ist die systematische Anwendung postkolonialer Konzepte und Fragestellungen auf die jeweiligen Gegenstandsbereiche. Daher ist es zunächst notwendig, diese Konzepte und Fragestellungen darzustellen, bevor anschließend auf die zu untersuchenden Gegenstandsbereiche eingegangen wird.

Die zentrale Fragestellung postkolonialer Ansätze ergibt sich bereits aus der Definition: Inwieweit sind Repräsentationen und Identitäten sowie damit verbundene materielle Praktiken auch nach der formellen Dekolonisierung noch durch den Prozess der Kolonialisierung geprägt? Hier handelt es sich mithin um die allgemeine Frage nach den Manifestationen von Kontinuitäten und Diskontinuitäten zwischen kolonialer und nachkolonialer Periode. Politikwissenschaftlich relevant wird diese Frage v. a. durch ihre Anwendung auf politische Institutionen (polity), politische Prozesse (politics) und konkrete Politikinhalte (policies).

9 Über die Gründe dafür ließe sich spekulieren: $\mathrm{Ob}$ dies an der vergleichsweise kurzen und wenig beachteten kolonialen Vergangenheit Deutschlands, an der (zumindest gegenüber den USA) ausgeprägteren kritischen Tradition in den Sozialwissenschaften, an der randständigen Position der Entwicklungsforschung oder an der (außerhalb des nationalsozialistischen Kontextes) seltenen Thematisierung von Rassismus hierzulande liegt, ist ungewiss. 
Weitere, präzisere Fragestellungen ergeben sich aus den Konzepten postkolonialer Theorien, auf die genauer einzugehen sein wird. Als wichtigste Konzepte der postkolonialen Theorien können m. E. die folgenden identifiziert werden: Orientalismus und Othering, Subalternität und Repräsentation, Hybridität, Provinzialisierung Europas. ${ }^{10}$ Die vier vorgestellten Konzepte sind in der postkolonialen Debatte mit einer Reihe von Kritikpunkten konfrontiert worden (für das Konzept des Orientalismus siehe z. B. Porter 1983; Ahmad 1992; Bhabha 1994, S. 101108; Loomba 1998, S. 43-51; Young 2001, S. 383-394; Frank 2004; Castro Varela u. Dhawan 2005, S. 29-54). Diese werden im vorliegenden Forschungsprogramm jedoch v. a. dann relevant, wenn sie sich auch in der empirischen Anwendung der Konzepte wiederfinden. Einige dieser Kritikpunkte können bereits durch Dialog und Interaktion zwischen den verschiedenen Konzepten angegangen werden. Beispielsweise wurde dem Konzept des Orientalismus seine binäre Logik vorgeworfen, die unterschiedliche Unterdrückungsverhältnisse jenseits des Kolonialismus nicht zu erfassen imstande scheint. Durch die gleichzeitige Anwendung des Subalternitätskonzepts wird jedoch ein Bewusstsein über die Mehrdimensionalität von Unterdrückungsverhältnissen gewährleistet.

\subsection{Orientalismus und Othering}

In seiner detaillierten Studie über die Darstellung des Orients in der abendländischen Kultur geht Edward Said davon aus, dass „der Orient keine unveränderliche Tatsache der Natur ist“, sondern „vom Menschen geschaffen“ (1981, S. 12), und zwar durch vielfältige Praktiken der Wissensproduktion (Reiseberichte, akademische Abhandlungen, aber auch Romane und andere literarische Werke), die eine soziale Wirklichkeit konstruieren, indem sie bestimmte Bilder, Vokabeln, Redeweisen und „Wahrheiten“ über den Orient und die Orientalen zur Verfügung stellen. In diesem Sinne spricht Said (recht ungenau an Foucault anknüpfend) vom Orientalismus als einem Diskurs (S. 8). Zentrales Merkmal dieses Diskurses ist nun laut Said, dass er „auf einer ontologischen und epistemologischen Unterscheidung basiert, die zwischen ,dem Orient' und ,dem Okzident ${ }^{\star}$ gemacht wurde" (S. 9). Diese Unterscheidung ermöglicht nicht nur die Homogenisierung und (meist negative) Stereotypisierung des Orients und seiner Bewohner, sondern erlaubt es auch „Europa (oder dem Westen) sich als dessen kontrastierendes Bild [...] zu definieren“ (S. 8) - als fortschrittlich gegenüber einem rückständigen, als rational gegenüber einem irrationalen, als liberal gegenüber einem despotischen Orient - und so eine „positionsbestimmte Überlegenheit“ (S. 15) zu erlangen. Vor diesem Hintergrund (und aufgrund zahlreicher verzerrter Darstellungen) kommt Said zu dem Schluss: „Der Orientalismus antwortete mehr der Kultur, die ihn produzierte, als einem mutmaßlichen Objekt“ (S. 32), m. a. W., das hervorgebrachte Wissen entsprach eher den Bedürfnissen des Westens als den Realitäten

10 Andere Konzepte wie Mimikry, Dritter Raum, Worlding, strategischer Essentialismus, Privilegien verlernen etc. wären ebenfalls denkbar, sollen an dieser Stelle allerdings vernachlässigt werden, weil sie außerhalb ihres Entstehungskontexts schwierig als abstrakte Analysestrategie zu fassen sind. 
des Ostens. Diese Bedürfnisse waren jedoch nicht nur identitärer, sondern auch politischer Art: Die gesellschaftliche Relevanz des Orientalismus bestand v. a. darin, dass er die koloniale Herrschaft über die Gebiete des Orients legitimierte, weshalb ihn Said auch als einen „Herrschaftsstil“ bzw. eine „Form des Autoritätsbesitzes“ über den Orient bezeichnet (S. 10).

Wenn wir nun von Saids konkretem Beispiel abstrahieren, können wir das Konzept des Orientalismus als die Wissensproduktion über die kulturell Anderen definieren (die ontologische Unterscheidung manifestiert sich nach der Diskreditierung des Rassendenkens überwiegend kulturell), die der Definition des eigenen Selbst dient und - dies ist v. a. für die Politikwissenschaft relevant - politische Ansprüche oder Ausschlüsse ermöglicht. Allgemein wird dies in den Kulturwissenschaften als „Othering“ bezeichnet - die Konstruktion eines Fremden, das als negative Projektionsfläche zur (Re-)Produktion einer positiven eigenen Identität dient. Reuter (2002) übersetzt Othering treffend mit „VerAnderung“. Diese Art der Wissensproduktion ist im Kolonialismus quasi omnipräsent gewesen und begegnet uns auch heute noch vielerorts. Stuart Hall hat im Anschluss an Said und Todorov (1999) herausgearbeitet, dass auch in der kolonialen Expansion Europas jenseits des Orients die Einordnung des Nicht-Westens in ein dichotomes, hierarchisches Repräsentationssystem zur Konstruktion der Identität des Westens als zivilisiert, rational, diszipliniert, überlegen usw. gedient hat (Hall 1994; siehe auch Melber 1992 und Nandy 2008). Von feministischer Seite ist zurecht darauf hingewiesen worden, dass diese dichotomen Zuschreibungen mit den Binäroppositionen maskulin-feminin und Kultur-Natur eng verbunden sind (z. B. Peterson 2003, S. 36).

Da es beim Orientalismus und dem abstrakteren, übergeordneten Othering um die Frage der Darstellung des Fremden und die damit verbundene Autorität geht und die Stimme der „VerAnderten“ nicht gehört und ihre mögliche Selbstdarstellung negiert wird, sind die Konzepte eng verknüpft mit den Fragen nach Subalternität und Repräsentation.

\subsection{Subalternität und Repräsentation}

Im vermutlich bekanntesten Text der Postkolonialen Studien setzt sich Gayatri Spivak mit der Frage auseinander: „Can the Subaltern speak?“ (Spivak 2008), mithin mit der Möglichkeit der Marginalisierten, selbst zu sprechen. Entgegen einer auch in den Sozialwissenschaften verbreiteten Meinung (Drekonja-Kornat 2004) reklamiert sie dabei nicht das Recht und die Fähigkeit der Unterdrückten zur Selbstartikulation, sondern problematisiert und negiert diese Möglichkeit.

Mit der Kategorie der Subalternen schließt Spivak zunächst an Gramsci an, der damit die unterdrückten Klassen bezeichnet hat. In der Rezeption der Subaltern Studies Group, zu der auch Spivak gehörte, verschiebt sich die Definition des Begriffs einerseits, um den Kontext des Kolonialismus und der internationalen Arbeitsteilung zu berücksichtigen: Als subaltern gelten all die gesellschaftlichen Gruppen, die weder auf nationaler noch auf regionaler und lokaler Ebene in das koloniale Herrschaftssystem eingebunden sind (Spivak 2008, S. 50), die also nicht über Zugeständnisse dazu gebracht wurden, die bestehende Hegemonie konsen- 
sual mit zu tragen. Andererseits erweist sich Subalternität auch als positional: „Dieselbe Klasse oder dasselbe Element, das in einer Gegend dominant war, [...] konnte in einer anderen zu den Dominierten gehören " (Guha zit. nach Spivak 2008, S. 50). Dies ermöglicht die Berücksichtigung mehrdimensionaler Herrschaftsverhältnisse, v. a. entlang der Linien Rasse, Klasse und Geschlecht: Personen können im Kontext des Kolonialismus hinsichtlich ihrer (stets konstruierten) Rasse unterdrückt, in den anderen Dimensionen aber gleichzeitig privilegiert sein. Diese Unterdrückung geht nun mit asymmetrischen Möglichkeiten der Repräsentation einher, sowohl im Sinne der symbolischen Repräsentation (Darstellung) als auch im Sinne der politischen Repräsentation (Vertretung). Spivak kommt nun (mit Marx) zu dem Schluss, dass bestimmte Gruppen darüber hinaus auch nicht über die Fähigkeit zur Selbst-Repräsentation verfügen, d. h. kein klares Bewusstsein über ihre wirklichen Interessen haben und diese artikulieren können: „Was die ,wahre' subalterne Gruppe angeht, [...] so gibt es hier kein nicht-repräsentierbares subalternes Subjekt, das selbst wissen und sprechen kann; die Lösung der Intellektuellen besteht nicht darin, sich der Repräsentation zu enthalten “ (Spivak 2008, S. 52). Hier (und an anderen Stellen) kritisiert sie Foucault und Deleuze stellvertretend für eine theoriepolitische Schule, die im Bewusstsein über die damit verbundenen Herrschaftsverhältnisse den Anspruch der Repräsentation der Subalternen zurückweist und behauptet, diese könnten für sich selbst sprechen - und so im Rekurs auf vermeintlich authentische Subjekte das Problem der Ideologie vollständig ausklammert.

Daher und eingedenk der Mehrdimensionalität der Unterdrückungsverhältnisse postuliert Spivak: „Wenn die Subalternen im Kontext kolonialer Produktion keine Geschichte haben und nicht sprechen können, dann ist die Subalterne als Frau sogar noch tiefer in den Schatten gedrängt. [...] Auf der anderen Seite der internationalen Arbeitsteilung angesiedelt, ist das ausgebeutete Subjekt nicht in der Lage, den Text weiblicher Ausbeutung zu erkennen und auszusprechen, selbst wenn nicht-repräsentierende Intellektuelle sich absurderweise dazu versteigen, diesen Frauen einen Raum zu schaffen, um selbst zu sprechen “ (2008, S. 57, 59). Spivak illustriert dies am Beispiel der Kontroverse um die gesetzliche Abschaffung des indischen Rituals der Witwenverbrennung unter der englischen Kolonialherrschaft: Die Frauen selbst kamen in dieser Kontroverse kaum zu Wort, und wenn doch, dann wurden ihre Äußerungen entweder von imperialistischer Seite als Beweis der Rückständigkeit der indischen Kultur und der befreienden Rolle der Kolonialherrschaft (wenn sie sich dagegen aussprachen) oder (im umgekehrten Fall) von patriarchaler Seite als Bekenntnis zur traditionellen Frauenrolle vereinnahmt: „Zwischen Patriarchat und Imperialismus, Subjektkonstitution und Objektformierung verschwindet die Figur der Frau [...] in eine gewaltförmige Pendelbewegung, die in der verschobenen Gestaltwerdung der zwischen Tradition und Modernisierung gefangenen ,Frau der Dritten Welt' besteht" (Spivak 2008, S. 101). Dass es bei den Witwenverbrennungen aufgrund des Erbrechts auch um politökonomische Konflikte ging, spielte in der Kontroverse keine Rolle (Spivak 2008, S. 87). Am Beispiel des gezielt symbolischen Selbstmordes einer mit ihr verwandten Aktivistin illustriert Spivak konkret die Ignoranz herrschender Diskurse gegenüber entsprechenden Artikulationsversuchen und folgert: „Die Subalterne als 
Frau kann nicht gehört oder gelesen werden. [...] Die Subalterne kann nicht sprechen “ (2008, S. 105-106). Diese apodiktische Schlussfolgerung hat Spivak später relativiert, ohne jedoch wichtige Elemente ihrer Argumentation zurückzunehmen (Spivak 1996, S. 287-308; 2008, S. 119-148).

Um die Konzepte Spivaks für Analysen politischer Strukturen und Phänomene produktiv anzuwenden, müssen wir uns demnach Folgendes vergegenwärtigen: Subalternität ist positional und mehrdimensional und verknüpft mit der unzureichenden Möglichkeit und Fähigkeit zur Selbstrepräsentation sowohl im symbolischen als auch im politischen Sinn. Die Konzepte anzuwenden hieße also zum einen nach der Positionierung eines Akteurs entlang der Unterdrückungslinien von Rasse, Klasse und Geschlecht zu fragen, nach seiner Subalternität in unterschiedlichen Kontexten. Zum anderen hieße es aber auch, die Frage nach der Handlungsfähigkeit der Subalternen zu stellen, wobei hier die Bedingungen, Praktiken und Hindernisse ihrer politischen Artikulation und Repräsentation zu analysieren sind, ohne einerseits auf die Autonomie authentischer Subjekte oder andererseits auf die Allmacht manipulativer Diskurse zu rekurrieren. Es geht demnach erstens darum, wer in welcher Hinsicht als subaltern bezeichnet werden kann und zweitens nicht nur darum, ob die Subalternen sprechen, sondern auch darum, wie (unter dem Einfluss welcher Diskurse) sie es tun, ob und wie (gefiltert durch welche Wahrnehmungsmuster und Strukturen der Öffentlichkeit) sie gehört werden, und ob und wie (abhängig von gesellschaftlichen Kräftekonstellationen) ihre Artikulationen politisch wirksam werden.

Wenn die Frage nach der Handlungsfähigkeit der Subalternen gestellt wird, so ist dies bereits eine gewisse Abkehr von der Vorstellung, die Kolonialisierung sei ein Prozess gewesen, in dem die Kolonisierenden aktiv und die Kolonisierten gänzlich passiv waren. Diese Abkehr stellt die Grundlage von Bhabhas Konzept der Hybridität dar.

\subsection{Hybridität}

Mit dem Begriff der Hybridität bezeichnet Bhabha nicht einfach (wie oftmals angenommen) einen Zustand der Vermischung zweier Kulturen, sondern ein etwas komplexeres spezifisches Resultat des Kolonialisierungsprozesses. Sein klassisches Beispiel für ein solches Resultat findet sich in dem Bericht eines indischen Katecheten Anfang des 19. Jahrhunderts vor dem Hintergrund der expliziten britischen Strategie, die einheimische Kultur und Religion durch die Verteilung von englischen Bibeln zu zersetzen (Bhabha 1994, S. 145-174). Dieser Katechet trifft außerhalb von Delhi unversehens auf eine Gruppe von Gläubigen, die sich zur gemeinsamen Bibellektüre versammelt haben, um das Wort Gottes (handschriftlich in Hindi übersetzt), das sie von einem Engel erhalten hätten, zu lesen und zu preisen. Auf die aufklärende Erläuterung des Katecheten, dass Gottes Wort schon vor langer Zeit den Europäern offenbart wurde und die Engländer die heilige Schrift in Indien verbreitet hätten, reagieren die Gläubigen mit unerschütterlicher Zurückweisung - „that cannot be, for they eat flesh“ (zit. nach Bhabha 1994, S. 146) - und es stellt sich heraus, dass die (streng vegetarischen) Einheimischen ihre ganz eigene Version vom Christentum entwickelt haben und sich von der religiö- 
sen Autorität des Katecheten nicht sehr beeindrucken lassen. Die Kolonialmacht ist also präsent, aber ambivalent: „split between its appearance as original and authoritative and its articulation as repetition and difference" (S. 153). Die Nachahmung des europäischen Glaubens auf eine „entstellte“ Art und Weise unterminiert die Autorität der Kolonialmacht, denn die negierte und unterdrückte Kultur (hier konkret der Vegetarismus) schreibt sich in die koloniale Präsenz (hier konkret die Bibel) wieder ein: „the trace of what is disavowed is not repressed but repeated as something different - a mutation, a hybrid“ (S. 159). Hybridität bezeichnet mithin die Produktivität kolonialer Diskurse, die aber zu einer strategischen Umkehrung der mit ihnen verbundenen Herrschaft durch Nichtanerkennung führt und die Identität der kolonialen Autorität destabilisiert: „other ,denied' knowledges enter upon the dominant discourse and estrange the basis of its authority" (S. 162). Im konkreten Beispiel hängt die Aneignung der christlichen Bibel mit ihrer Instrumentalisierung gegen das indische Kastensystem zusammen, mit ihrer Rolle als potenzielle Waffe im Kampf gegen traditionelle Unterdrückungsverhältnisse. Gleichzeitig hat sie sich emanzipiert von der „mission civilisatrice“, die mit der kolonialen Unternehmung zusammenhing, da der göttliche Status des Buches mit einer europäischen Herkunft als schlichtweg inkompatibel empfunden wird („how can the word of God come from the flesh-eating mouths of the English?", S. 166). Die koloniale Strategie der Bibelverbreitung hatte demnach zwar deutliche Effekte auf die kolonisierte Gesellschaft, deren Art entsprach jedoch keineswegs immer der ursprünglichen Intention, sondern führte zur entstellenden Wiederholung der kolonialen Präsenz und zur Infragestellung ihrer Autorität. Dies ist laut Bhabha eine notwendige Begleiterscheinung der entsprechenden kolonialen Strategien.

Bhabha verdeutlicht mit seinem Konzept, dass der Prozess der Kolonialisierung sowohl das kolonisierende Selbst als auch das kolonisierte Andere transformiert und binäre Zuschreibungen, die die aktive Rolle in diesem Prozess nur der einen Seite zuweisen, verfehlt sind. Wenn das Konzept der Hybridität nun produktiv angewendet werden soll, ist es allgemeiner und abstrakter als eine Strategie der Detotalisierung von Herrschaft zu fassen. Diese lässt sich in dreierlei Fragestellungen fassen: die Frage nach den Grenzen und Instabilitäten von Herrschaft, die Frage nach den nichtintendierten Effekten von Machtausübung und die Frage nach der subversiven Aneignung und Verfremdung von herrschenden Diskursen. In dieser Hinsicht stellt es eine notwendige und sinnvolle Ergänzung der Konzepte von Orientalismus und Othering dar: Während diese die Konstruktion des Anderen im Diskurs und die damit einhergehende Prägung von Identitäten untersuchen, vertritt das Konzept der Hybridität die These, dass solche Konstruktionen und Prägungen keineswegs einheitlich und total sind, dass es durch die Übertragung in verschiedene lokale Kontexte stets zu Bedeutungsverschiebungen und Aneignungsprozessen kommt, dass Widerstand auch in der Form einer bestimmten Art der Unterwerfung vorkommen kann, und dass Herrschaft nie so bruchlos und stabil ist, wie sie vorgibt. 


\subsection{Provinzialisierung Europas}

Ein viertes Konzept, das in seiner allgemeinen Version ein Querschnittsthema vieler postkolonialer Untersuchungen ist, hat der indische Historiker Dipesh Chakrabarty geprägt, als er von der „Provinzialisierung Europas“ sprach (Chakrabarty 2000, 2002). Sein Ausgangspunkt ist die Erkenntnis, dass in der Geschichtswissenschaft, aber auch in den anderen Geistes- und Gesellschaftswissenschaften „Europa“ immer noch das souveräne, theoretische Subjekt" sei (2002, S. 283), das die entsprechende Betrachtungsweise nachhaltig präge. Über die Kritik an einer „asymmetrischen Unkenntnis“ (nicht-westliche HistorikerInnen müssen im Wissenschaftsbetrieb die Arbeit der westlichen kennen aber nicht umgekehrt) hinaus formuliert Chakrabarty: „Seit Generationen haben Philosophen und Sozialwissenschaftler Theorien aufgestellt, welche für die gesamte Menschheit Gültigkeit beanspruchen. Formuliert wurden diese Aussagen allerdings [...] in relativer und bisweilen absoluter Unkenntnis der Erfahrungen der Mehrheit der Menschheit, das heißt derjenigen Menschen, die in nichtwestlichen Kulturen leben“ (S. 284). Trotz dieser Perspektivität spricht er diesen Theorien jedoch nicht ihren Wert ab: „Das alltägliche Paradox der Sozialwissenschaften in der Dritten Welt besteht darin, dass wir diese Theorien ungeachtet ihrer Unkenntnis, unserer' Erfahrungen für das Verständnis unserer Gesellschaften außerordentlich nützlich finden“" (S. 284, Hervorhebung im Original).

Dennoch habe der kategoriale Eurozentrismus dieser Theorien vielerorts beschränkende Wirkung entfaltet, nicht zuletzt im Hinblick auf die antikolonialen Bewegungen: Diese haben in der Auseinandersetzung mit der Kolonialherrschaft einerseits „Elemente eines kollektiven Gedächtnisses mobilisiert [...], die sowohl antihistorisch als auch antimodern waren“, taten dies andererseits aber „im Kontext ,moderner' Institutionen und teilweise zugunsten des modernisierenden Projekts des Nationalismus“ (S. 302). Die in seinen Augen kurzsichtige Fixierung auf "den Nationalstaat als die wünschenswerteste Form politischer Gemeinschaft" ( $S$. 304) sieht Chakrabarty als den gemeinsamen Nenner von europäischem Imperialismus und antikolonialem Widerstand. Hier manifestiere sich eine Identifizierung von „Moderne“ und „Europa“, die nicht nur das Werk von Europäern sei und der er sein „Projekt der Provinzialisierung Europas“ entgegensetzen möchte (S. 304-306). In diesem ginge es keinesfalls um eine Zurückweisung der Moderne aus der Position eines kulturellen Relativismus, aber um eine Transzendierung der mit dem Liberalismus einhergehenden Konstruktionen von Staat, Staatsbürgerschaft und Privatsphäre (S. 305), um so Europa als wichtigen Teil, aber nicht mehr als ausschließliches Subjekt der Geschichte und alleinige Inkarnation der Moderne zu sehen und die entsprechenden Begriffe als partikularen und nicht universellen Ursprungs zu erkennen. „Den Versuch zu unternehmen, dieses ,Europa' zu provinzialisieren, heißt, das Moderne als ein Feld von Auseinandersetzungen zu begreifen. Es bedeutet, die bislang privilegierten Erzählungen der Staatsbürgerschaft mit Erzählungen anderer menschlicher Bindungen zu überschreiben, die sich von erträumten Vergangenheiten und Zukunftsentwürfen nähren, in denen Kollektivitäten weder durch die Rituale von Staatsbürgerschaft noch durch den Alptraum der durch die ,Moderne‘ geschaffenen ,Tradition“ definiert sind“ (S. 309). 
Wenn es nun um eine Anwendung des Konzepts jenseits dieser etwas wolkigen Formulierungen geht, so lässt sich der Imperativ formulieren, den Eurozentrismus bestehender Kategorien der Sozialwissenschaften, bzw. allgemeiner formuliert die Kontingenz, Perspektivität und Partikularität vermeintlich universeller Begriffe herauszuarbeiten, um Raum zu schaffen für alternative Konstruktionen und Praktiken. Dabei darf es jedoch gerade nicht um einen Antimodernismus oder eine pauschale Zurückweisung der westlichen Kultur unter Bezug auf konstruierte und essenzialisierte eigene Traditionen oder Kulturen gehen. Zumindest implizit wird mit dem Konzept der Provinzialisierung Europas ein Kernanliegen der meisten postkolonialen Arbeiten formuliert: darauf aufmerksam zu machen, dass die in den Sozialwissenschaften vorherrschenden Sichtweisen i. d. R. nicht einfach neutral, wissenschaftlich und kulturunabhängig sind, mithin nach ihrer westlichen Prägung und der Möglichkeit alternativer, nicht eurozentrischer Wissensformen zu fragen.

Zusammenfassend seien die Fragestellungen der postkolonialen Konzepte noch einmal vereinfacht und stichwortartig dargestellt:

\section{Tabelle 1: Fragestellungen postkolonialer Konzepte}

\begin{tabular}{|l|l|}
\hline Theorieansatz & Fragestellungen beziehen sich auf: \\
\hline $\begin{array}{l}\text { Postkoloniale Studien allge- } \\
\text { mein }\end{array}$ & $\begin{array}{l}\text { Koloniale Kontinuitäten und Diskontinuitäten von Repräsen- } \\
\text { tationen, Identitäten und Praktiken }\end{array}$ \\
\hline Orientalismus/Othering & $\begin{array}{l}\text { Wissensproduktion über Andere in Verbindung mit eigener } \\
\text { Identität und politischen Ansprüchen/Ausschlüssen }\end{array}$ \\
\hline Subalternität/Repräsentation & $\begin{array}{l}\text { Positionierung in mehrdimensionalen Unterdrückungsverhält- } \\
\text { nissen, Bedingungen der politischen Artikulation und Reprä- } \\
\text { sentation }\end{array}$ \\
\hline Hybridität & $\begin{array}{l}\text { Grenzen und nichtintendierte Effekte von Herrschaft, subver- } \\
\text { sive Aneignung und Umformung herrschender Diskurse }\end{array}$ \\
\hline Provinzialisierung Europas & $\begin{array}{l}\text { Eurozentrismus bzw. Partikularität vermeintlich universeller } \\
\text { Begriffe und mögliche Alternativen }\end{array}$ \\
\hline
\end{tabular}

\subsection{Eine Postkoloniale Politikforschung}

Wie sind diese Fragestellungen nun konkret auf politikwissenschaftliche Gegenstandsbereiche anzuwenden? Für eine ausführliche Darstellung entsprechender Forschungspraxis fehlt hier der Raum. Einige allgemeine Anmerkungen sind jedoch an dieser Stelle möglich: Hinsichtlich der Methoden der postkolonialen Politikforschung sind eingedenk der oben formulierten Konzepte zwei Schwerpunkte zu setzen. Ein methodischer Schwerpunkt liegt hierbei auf der Analyse der wechselseitigen Konstituierung von Identitäten, Repräsentationssystemen und politischen Praktiken und Institutionen (vgl. Peterson 2003). Ein anderer Schwerpunkt besteht in der unverzichtbaren Berücksichtigung der Artikulationen der Betroffenen, ohne sie dabei jedoch als authentische Stimme zu verklären. Aus postkolonialer Perspektive ist eine dialogische Forschung anzustreben, in der Subjekte im Süden nicht Objekte, sondern KoproduzentInnen des Wissens sind. Dies impliziert zum einen eine Forschungspraxis, die nicht nur die Rolle der Forschenden 
reflektiert, sondern auch für die Intervention der anderen beteiligten Personen in die Forschungssituation Raum lässt (vgl. Ploder 2009). Zum anderen impliziert es, die Ergebnisse der Forschung den an ihrer Generierung beteiligten Gruppen zu präsentieren und mit ihnen zu diskutieren.

Davon abgesehen bedient sich die postkoloniale Politikforschung des üblichen Methodenrepertoires primär der qualitativen Sozialforschung (v. a. Interviews, teilnehmende Beobachtung, oral history für die Datenerhebung; v. a. hermeneutische Inhalts- und Diskursanalyse für die Datenanalyse). Quantitative Methoden können jedoch auch eingesetzt werden, z. B. im Rahmen der Analyse größerer Textcorpora oder im Hinblick auf ökonomische Fragen.

Vier Gegenstandsbereiche der Politikwissenschaft lassen sich als für eine postkoloniale Analyse naheliegend identifizieren, da sie mit nachkolonialen Gesellschaften oder mit Nord-Süd-Beziehungen befasst sind. Folgende Forschungsfragen skizzieren mögliche Konturen einer postkolonialen Politikforschung in diesem Bereich:

Entwicklungszusammenarbeit (EZ) und Entwicklungspolitik: Hier wäre zu fragen, inwiefern die Wahrnehmung von Menschen im Süden durch Akteure der EZ immer noch von kolonialen Steretypen geprägt ist bzw. inwiefern sich diese Wahrnehmung durch die Prinzipien der Pariser Deklaration (wie z. B. ownership) verändert. Wo werden Hierarchien gegenüber ,zu Entwickelnden' mit der vermeintlichen Überlegenheit technischen Wissens und eines ,fortschrittlicheren“ Gesellschaftsmodells gerechtfertigt, und inwieweit sind hier auch Subjekte aus dem Süden die Akteure? Sind Good-Governance-Auflagen in der EZ menschenrechtlich geboten oder ,neokoloniale‘ Eingriffe in die Souveränität ehemaliger Kolonien? Warum wird das eurozentrische Konstrukt der ,Entwicklung' weiter reproduziert und wie sähen Alternativen dazu aus? Lässt sich im Anschluss an eine entsprechende konzeptionelle „Provinzialisierung Europas“ eine neue universelle Norm positiver Gesellschaftsveränderung finden oder müssen solche Normen lokal sein? Wie manifestiert sich der Ethnozentrismus neuer Geberländer wie China?

Internationale politische Ökonomie und global economic governance: Wie verändern sich Wahrnehmungsmuster des Südens durch Diskontinuitäten in der internationalen Arbeitsteilung, Finanzkrisen oder den Aufstieg der chinesischen Volkswirtschaft? Wird die ungleiche Stimmenverteilung in den Bretton-WoodsInstitutionen unter Rückgriff auf Konstruktionen des Anderen gerechtfertigt? Hat sich die Praxis von Weltbank und IWF gegenüber Subalternen durch den gestiegenen Anteil ihres Personals aus ehemaligen Kolonien oder durch die partizipative Umgestaltung der Strukturanpassungsprogramme verändert? Wo dienen altbewährte Stereotypen zur Erklärung von ökonomischer Ungleichheit im Weltmaßstab? Inwiefern sind die WTO-Regeln vergleichbar mit dem ,Freihandelsimperialismus' des Britischen Empire? Und im Gegenzug: Inwiefern dienen Vorwürfe des ,Neokolonialismus' nationalistischen Regierungen im Süden als Legitimationsressource oder Ausweichmanöver gegenüber Forderungen nach Sozialstandards?

Friedens- und Konfliktforschung und Sicherheitspolitik: Lassen sich in den Begründungen für ,humanitäre Interventionen' und ,neue Protektorate' erneut koloniale Argumentationsmuster auffinden? (Der von Spivak [2008] als Standardbegründung identifizierte Satz „white men saving brown women from brown men“ 
war z. B. durchaus relevant in den Debatten um den Afghanistan-Krieg.) Inwiefern spielen z. B. Stereotypen der, orientalischen Despotie'in Fragen der Weiterverbreitung von Atomwaffen hinein? Wie ist der Zusammenhang zwischen der wahrgenommenen Gefährlichkeit von Sprengköpfen und der Kultur ihrer Besitzer? Haben politische Entscheidungen der Kolonialherrschaft und damit verknüpfte Identitätsprägungen auch heute noch Auswirkungen auf interne Konflikte in nachkolonialen Gesellschaften? Wie ermöglicht die Konstruktion der ,gefährlichen Anderen' die Herausbildung einer militanten christlichen europäischen Identität bei bestimmten politischen Gruppen?

Migration und Migrationspolitik: Prozesse des Othering gegenüber MigrantInnen sind in ihrer Dynamik zu untersuchen, beispielsweise wenn neuerdings im Rahmen der Einbürgerung Bekenntnisse zur Akzeptanz von Homosexualtät verlangt werden. Welche Arten von Migration und welche MigrantInnen werden als Bedrohung wahrgenommen, welche als wirtschaftliche Notwendigkeit für ,uns'? Lässt sich Arbeitsmarktpolitik gegenüber MigrantInnen als „Inversion kolonialer Expansionsformen“ ( $\mathrm{Ha})$ interpretieren und ermöglicht Othering intensivierte Aneignung von Produktivkraft? Inwiefern repräsentieren migrantische Organisationen die Subalternen? Wo werden Konstruktionen des migrantischen Anderen von Betroffenen angeeignet und umgedeutet (z. B. „Kanak Attak“)? Inwiefern werden Praktiken der Migrationssteuerung durch migrantische Akteure unterlaufen („Autonomie der Migration“)?

Dies sind nur wenige Beispiele für Forschungsfragen, die sich aus der Anwendung postkolonialer Ansätze auf politikwissenschaftliche Gegenstandsbereiche ergeben können. Auch sie untermauern m. E. folgendes Fazit: Die innovativen theoretischen Ansätze der Postkolonialen Studien würden von einer stärkeren Verknüpfung mit systematischer empirischer Politikforschung ebenso profitieren wie die erwähnten Teilbereiche der Politikwissenschaft. Die hier skizzierte Vorgehensweise einer Anwendung postkolonialer Analysestrategien auf diese Gegenstandsbereiche stellt somit, so die hier vertretene These, ein produktives und innovatives Forschungsprogramm dar.

\section{Literatur}

Abrahamsen, Rita. 2000. Disciplining Democracy. Development Discourse and Good Governance in Africa. London: Zed Books.

Agathangelou, Anna M. 2004. 'Sexing' Globalization in International Relations: Migrant

Sex and Domestic Workers in Cyprus, Greece and Turkey. In Power, Postcolonialism and International Relations. Reading Race, Gender and Class, Hrsg. Geeta Chowdhry und Sheila Nair, 142-169. London: Routledge.

Agathangelou, Anna M. 2006. The Global Political Economy of Sex. Desire, Violence and Insecurity in Mediterranean Nation States. London: Palgrave Macmillan.

Agathangelou, Anna M., und Lily H. M. Ling. 2004. Power, Borders, Security, Wealth: Lessons of Violence and Desire from September 11. International Studies Quarterly 48:517-538.

Agathangelou, Anna M., und Lily H. M. Ling. 2009. Transforming World Politics. From Empire to Multiple Worlds. London: Routledge. 
Ahmad, Aijaz. 1992. Orientalism and After. In Colonial Discourse and Post-Colonial Theory. A Reader, Hrsg. Patrick Williams und Laura Chrisman, 162-171. New York: Columbia University Press.

Ashcroft, Bill, Gareth Griffiths und Helen Tiffin (Hrsg.). 1995. The Post-Colonial Studies Reader. London: Routledge.

Bhabha, Homi. 1990. Nation and Narration. London: Routledge.

Bhabha, Homi. 1994. The Location of Culture. London: Routledge.

Biswas, Shampa. 2001. 'Nuclear Apartheid' as Political Position: Race as Postcolonial Resource? Alternatives 26:485-522.

Blaut, James M. 1993. The Colonizer's Model of the World. Geographic Diffusionism and Eurocentric History. New York: Guilford Press.

Castro Varela, Maria do Mar, und Nikita Dawahn. 2005. Postkoloniale Theorie. Eine kritische Einführung. Bielefeld: transcript.

Chakrabarty, Dipesh. 2000. Provincialising Europe. Postcolonial Thought and Historical Difference. Princeton: Princeton University Press.

Chakrabarty, Dipesh. 2002. Europa provinzialisieren. Postkolonialität und die Kritik der Geschichte In Jenseits des Eurozentrismus. Postkoloniale Perspektiven in den Geschichts- und Kulturwissenschaften, Hrsg. Sebastian Conrad und Shalini Randeria, 283-312. Frankfurt a. M.: Campus Verlag.

Chojnacki, Sven. 2004. Wandel der Kriegsformen? Ein kritischer Literaturbericht. Leviathan 32:402-424.

Chowdhry, Geeta. 2004. Postcolonial Interrogations of Child Labor: Human Rights, Carpet Trade, and Rugmark in India. In Power, Postcolonialism and International Relations. Reading Race, Gender and Class, Hrsg. Geeta Chowdhry und Sheila Nair, 225253. London: Routledge.

Chowdhry, Geeta, und Sheila Nair (Hrsg.). 2004. Power, Postcolonialism and International Relations. Reading Race, Gender and Class. London: Routledge.

Conrad, Sebastian, und Shalini Randeria (Hrsg.). 2002. Jenseits des Eurozentrismus. Postkoloniale Perspektiven in den Geschichts- und Kulturwissenschaften. Frankfurt a. M.: Campus Verlag.

Cooper, Frederick, und Randall Packard (Hrsg.). 1997. International Development and the Social Sciences. Essays on the History and Politics of Knowledge. Berkeley: University of California Press.

Costa, Sergio. 2005. Postkoloniale Studien und Soziologie: Differenzen und Konvergenzen. Berliner Journal für Soziologie 15:283-292.

Cowen, Michael, und Robert Shenton. 1996. Doctrines of Development. London: Routledge.

Crush, Jonathan (Hrsg.). 1995. Power of Development. London: Routledge.

Darby, Philip (Hrsg.). 2000. At the Edge of International Relations. Postcolonialism, Gender and Dependency. London: Continuum.

Darby, Philip, und Albert J. Paolini. 1994. Bridging International Relations and Postcolonialism. Alternatives 19:371-397.

Daase, Christopher. 2003. Krieg und politische Gewalt: Konzeptionelle Innovationen und theoretischer Fortschritt. In Die neuen Internationalen Beziehungen. Forschungsstand und Perspektiven in Deutschland, Hrsg. Gunter Hellmann, Klaus Dieter Wolf und Michael Zürn, 163-208. Baden-Baden: Nomos. 
Dhawan, Nikita. 2009. Zwischen Empire und Empower: Dekolonisierung und Demokratisierung. femina politica 18:52-63.

Dirlik, Arif. 1994. The Postcolonial Aura: Third World Criticism in the Age of Global Capitalism. Critical Inquiry 20:328-356.

Drekonja-Kornat, Gerhard. 2004. Wer darf wie über die Dritte Welt schreiben? Leviathan 32:431-439.

Doty, Roxanne Lynn. 1996. Imperial Encounters. The Politics of Representation in NorthSouth Relations. Minneapolis: University of Minnesota Press.

Eriksson Baaz, Maria. 2005. The Paternalism of Partnership. A Postcolonial Reading of Identity in Development Aid. London: Zed Books.

Escobar, Arturo. 1995. Encountering Development. The Making and Unmaking of the Third World. Princeton: Princeton University Press.

Esteva, Gustavo. 1987. Regenerating People's Space. Alternatives 12:125-152.

Frank, Michael C. 2004. Kolonialismus und Diskurs: Michel Foucaults ,Archäologie‘ in der postkolonialen Theorie. In PostModerne DeKonstruktionen. Ethik, Politik und Kultur am Ende einer Epoche, Hrsg. Susanne Kollmann und Kathrin Schödel, 139156. Münster: Lit.

Gandhi, Leela. 1998. Postcolonial Theory: A Critical Introduction. Edinburgh: Edinburgh University Press.

Gathii, James Thuo. 2006. Dispossession Through International Law: Iraq in Historical and Comparative Context. In Decolonizing International Relations, Hrsg. Branwen Gruffydd Jones, 131-154. Lanham: Rowman \& Littlefield.

George, Susan. 1988. A Fate Worse Than Debt. A Radical New Analysis of the Third World Debt Crisis. Harmondsworth: Penguin.

Gills, Barry, Joel Rocamora und Richard Wilson (Hrsg.). 1993. Low Intensity Democracy. Political Power in the New World Order. London: Pluto.

Golly, Nadine, und Stephan Cohrs (Hrsg.). 2008. deplatziert! Interventionen postkolonialer Kritik. Berlin: WVB.

Goudge, Paulette. 2003. The Whiteness of Power. Racism in Third World Development and Aid. London: Lawrence \& Wishart.

Grovogui, Siba N. 2007. Postcolonialism. In International Relations Theories. Discipline and Diversity, Hrsg. Tim Dunne, Milja Kurki und Steve Smith, 229-246. Oxford: Oxford University Press.

Gruffydd Jones, Branwen (Hrsg.). 2006a. Decolonizing International Relations. Lanham: Rowman \& Littlefield.

Gruffydd Jones, Branwen. 2006b. Introduction: International Relations, Eurocentrism, and Imperialism. In: Decolonizing International Relations, Hrsg. ders., 1-22. Lanham: Rowman \& Littlefield.

Ha, Kien Nghi. 1999. Ethnizität und Migration. Münster: Westfälisches Dampfboot.

Ha, Kien Nghi. 2004. Ethnizität und Migration Reloaded. Kulturelle Identität, Differenz und Hybridität im postkolonialen Diskurs. Berlin: WVB.

Ha, Kien Nghi, Nicola Lauré al-Samarai und Sheila Mysorekar (Hrsg.). 2007. relvisionen. Postkoloniale Perspektiven von People of Color auf Rassismus, Kulturpolitik und Widerstand in Deutschland. Münster: Unrast.

Hall, Stuart. 1994. Der Westen und der Rest: Diskurs und Macht. In Rassismus und kulturelle Identität. Ausgewählte Schriften 2, Hrsg. ders., 137-179. Hamburg: Argument. 
Hall, Stuart. 2002. Wann gab es ,das Postkoloniale'? Denken an der Grenze. In Jenseits des Eurozentrismus. Postkoloniale Perspektiven in den Geschichts- und Kulturwissenschaften, Hrsg. Sebastian Conrad und Shalini Randeria, 219-246. Frankfurt a. M.: Campus Verlag.

Hippler, Jochen (Hrsg.). 1994. Demokratisierung der Machtlosigkeit. Politische Herrschaft in der Dritten Welt. Hamburg: Konkret.

Kahl, Martin, und Ulrich Teusch. 2004. Sind die ,neuen Kriege' wirklich neu? Leviathan 32:382-401.

Kapoor, Ilan. 2008. The Postcolonial Politics of Development. London, Routledge.

Kerner, Ina. 1999. Feminismus, Entwicklungszusammenarbeit und Postkoloniale Kritik. Eine Analyse von Grundkonzepten des Gender-and-Development Ansatzes. Hamburg: Lit.

Kerner, Ina. 2009. Differenzen und Macht. Zur Anatomie von Rassismus und Sexismus. Frankfurt a. M.: Campus Verlag.

Kothari, Uma. 2005. From Colonial Administration to Development Studies: A Postcolonial Critique of the History of Development Studies. In A Radical History of Development Studies. Individuals, Institutions, Ideologies, Hrsg. ders., 47-66. London: Zed Books.

Kothari, Uma. 2006a. Critiquing 'Race' and Racism in Development Discourse and Practice. Progress in Development Studies 6:1-7.

Kothari, Uma. 2006b. An Agenda for Thinking About 'race' in Development. Progress in Development Studies 6: 9-23.

Lapid, Yosef. 1989. The Third Debate: On the Prospects of International Theory in a PostPositivist Era. International Studies Quarterly 33:235-254.

Ling, Lily H. M. 2002. Postcolonial International Relations. Conquest and Desire between Asia and the West. Basingstoke: Palgrave Macmillan.

Ling, Lily H. M. 2004. Cultural Chauvinism and the Liberal International Order: 'West versus Rest' in Asia's Financial Crisis. In Power, Postcolonialism and International Relations. Reading Race, Gender and Class, Hrsg. Geeta Chowdhry und Sheila Nair, 115141. London: Routledge.

Loomba, Ania. 1998. Colonialism/Postcolonialism. London: Routledge.

Lossau, Julia. 2002. Die Politik der Verortung. Eine postkoloniale Reise zu einer, anderen Geografie. Bielefeld: Transcript.

Mbembe, Achille. 2001. On the Postcolony. Berkeley: University of California Press.

McEwan, Cheryl. 2009. Postcolonialism and Development. London: Routledge.

Melber, Henning. 1992. Der Weisheit letzter Schluß. Rassismus und kolonialer Blick. Frankfurt a. M.: Brandes \& Apsel.

Menzel, Ulrich. 1992. Das Ende der Dritten Welt und das Scheitern der großen Theorie. Frankfurt a. M.: Suhrkamp.

Menzel, Ulrich. 1993. 40 Jahre Entwicklungsstrategie = 40 Jahre Wachstumsstrategie. In Handbuch der Dritten Welt. Band I: Grundprobleme, Theorien, Strategien, Hrsg. Dieter Nohlen und Franz Nuscheler, 131-155. Bonn: Dietz.

Menzel, Ulrich. 2010. Entwicklungstheorie. In Entwicklungspolitik. Theorien - Probleme - Strategien, Hrsg. Reinhard Stockmann, Ulrich Menzel und Franz Nuscheler, 11-160. Stuttgart: Oldenbourg. 
Merkel, Wolfgang. 1999. Systemtransformation. Eine Einführung in die Theorie und Empirie der Transitionsforschung. Wiesbaden: VS Verlag für Sozialwissenschaften.

Meyer, Jörg. 2008. The Concealed Violence of Modern Peace(-Making). Millennium 36:555-574.

Mies, Maria, und Vandana Shiva. 1993. Ecofeminism. London: Zed Books.

Moore, David, und Gerald J. Schmitz (Hrsg.). 1995. Debating Development Discourse. Institutional and Popular Perspectives. Basingstoke: Palgrave Macmillan.

Moses, A. Dirk, und Dan Stone (Hrsg.). 2006. Colonialism and Genocide. London: Routledge.

Mosley, Paul, Jane Harrigan und John Toye. 1991. Aid and Power. The World Bank and Policy-based Lending. London: Routledge.

Mosse, David, und David Lewis. 2005. The Aid Effect. Giving and Governing in International Development. London: Pluto.

Münkler, Herfried. 2002. Die neuen Kriege. Bonn: Bundeszentrale für politische Bildung.

Murray Li, Tania. 2007. The Will to Improve. Governmentality, Development, and the Practice of Politics. Durham: Duke University Press.

Nair, Sheila. 2004. Human Rights and Postcoloniality: Representing Burma. In Power, Postcolonialism and International Relations. Reading Race, Gender and Class, Hrsg. Geeta Chowdhry und Sheila Nair, 254-284. London: Routledge.

Nandy, Ashis. 1992. Traditions, Tyranny, and Utopias. Essays in the Politics of Awareness. Delhi: Oxford University Press.

Nandy, Ashis. 2008. Der Intimfeind. Verlust und Wiederaneignung der Persönlichkeit im Kolonialismus. Nettersheim: Verlag Graswurzelrevolution.

Peterson, Spike. 2003. A Critical Rewriting of Global Political Economy. Integrating Reproductive, Productive and Virtual Economies. London: Routledge.

Ploder, Andrea. 2009. Wollen wir uns irritieren lassen? Für eine Sensibilisierung der Methoden qualitativer Sozialforschung zur interkulturellen Kommunikation durch postkoloniale Theorie. Forum Qualitative Sozialforschung 10, Art. 42. http://www.qualitative-research.net/index.php/fqs/article/view/1232/2679. Zugegriffen 24.3.2011.

Plumelle-Uribe, Rosa Amelia. 2004. Weiße Barbarei: Vom Kolonialrassismus zur Rassenpolitik der Nazis. Zürich: Rotpunktverlag.

Porter, Dennis. 1983. Orientalism and its Problems. In Colonial Discourse and Post-Colonial Theory. A Reader, Hrsg. Patrick Williams und Laura Chrisman, 150-161. New York: Columbia University Press.

Purkarthofer, Petra. 2009. Rassismus, Maskulinismus und Eurozentrismus als materielle Praxen postkolonialer Hegemonie. In Globalisierung, Macht und Hegemonie. Perspektiven einer kritischen Internationalen Politischen Ökonomie, Hrsg. Eva Hartmann, Caren Kunze und Ulrich Brand, 43-69. Münster: Westfälisches Dampfboot.

Rahnema, Majid (Hrsg.). 1997. The Post-Development Reader. London: Zed Books.

Randeria, Shalini, und Andreas Eckert (Hrsg.). 2009. Vom Imperialismus zum Empire. Frankfurt a. M.: Suhrkamp.

Razack, Sherene H. 2004. Dark Threats \& White Knights. The Somalia Affair, Peacekeeping, and the New Imperialism. Toronto: University of Toronto Press.

Reuter, Julia. 2002. Ordnungen des Anderen. Zum Problem des Eigenen in der Soziologie des Fremden. Bielefeld: transcript. 
Reuter, Julia, und Matthias Wieser. 2006. Postcolonial, gender und science studies als Herausforderung der Soziologie. Soziale Welt 57:177-192.

Reuter, Julia, und Paula-Irene Villa (Hrsg.). 2009. Postkoloniale Soziologie. Empirische Befunde, theoretische Anschlïsse, politische Intervention. Bielefeld: transcript.

Sachs, Wolfgang (Hrsg.). 1993. Wie im Westen so auf Erden. Ein polemisches Handbuch zur Entwicklungspolitik. Reinbek: Rowohlt.

Said, Edward. (1978) 1981. Orientalismus. Frankfurt a. M.: Ullstein.

Said, Edward. (1993) 1994. Kultur und Imperialismus. Einbildungskraft und Politik im Zeitalter der Macht. Frankfurt a. M.: Fischer.

Schlichte, Klaus. 2002. Neues über den Krieg? Einige Anmerkungen zum Stand der Kriegsforschung in den Internationalen Beziehungen. Zeitschrift für Internationale Beziehungen 9:112-138.

Schlichte, Klaus. 2006. Neue Kriege oder alte Thesen? Wirklichkeit und Repräsentation kriegerischer Gewalt in der Politikwissenschaft. In Neue Kriegstheorien, Hrsg. Anna Geiss, 111-131. Baden-Baden: Nomos.

Schubert, Gunter, und Rainer Tetzlaff (Hrsg.). 1998. Blockierte Demokratien in der Dritten Welt. Opladen: Leske + Budrich.

Shohat, Ella. 1992. Notes on the 'Post-Colonial'. Social Text 10:99-113.

Sidaway, James. 2007. Spaces of Postdevelopment. Progress in Human Geography 31: 346-361.

Spivak, Gayatri Chakravorty. 1990. The Post-colonial Critic. Interviews, Strategies, Dialogues. Hrsg. Sarah Harasym. London: Routledge.

Spivak, Gayatri Chakravorty. 1996. The Spivak Reader. Hrsg. Donna Landry und Gerald Maclean. London: Routledge.

Spivak, Gayatri Chakravorty. (1988) 2008. Can the Subaltern Speak? In dies.: Can the Subaltern Speak? Postkolonialität und subalterne Artikulation, 17-118. Wien: Turia+Kant.

Steyerl, Hito, und Encarnacion Gutierrez Rodriguez (Hrsg.). 2003. Spricht die Subalterne deutsch? Migration und postkoloniale Kritik. Münster: Unrast.

Sylvester, Christine. 1999. Development Studies and Postcolonial Studies: Disparate Tales of the 'Third World'. Third World Quarterly 20:703-721.

Teivainen, Teivo. 2009. Globalisierung der ökonomischen Überwachung: Der Internationale Währungsfonds als moderner Priester. In Vom Imperialismus zum Empire, Hrsg. Shalini Randeria und Andreas Eckert, 105-136. Frankfurt a. M.: Suhrkamp.

Todorov, Tzvetan. 1999. The Conquest of America. The Question of the Other. Norman: University of Oklahoma Press.

White, Sarah. 2002. Thinking Race, Thinking Development. Third World Quarterly 23:407-419.

Williams, Patrick, und Laura Chrisman (Hrsg.). 1994. Colonial Discourse and Post-Colonial Theory. A Reader. New York: Columbia University Press.

Young, Robert. 2001. Postcolonialism. A Historical Introduction. Oxford: Blackwell.

Young, Robert. 2003. Postcolonialism. A Very Short Introduction. Oxford: Oxford University Press.

Ziai, Aram. 2004. Entwicklung als Ideologie? Das klassische Entwicklungsparadigma und die Post-Development Kritik. Ein Beitrag zur Analyse des Entwicklungsdiskurses. Hamburg: Deutsches Übersee-Institut. 
Ziai, Aram. 2006. Zwischen Global Governance und Post-Development: Entwicklungspolitik aus diskursanalytischer Perspektive. Münster: Westfälisches Dampfboot.

Ziai, Aram. 2007. Exploring Post-Development: Theory and Practice, Problems and Perspectives. London: Routledge.

Ziai, Aram. 2010. From Development Discourse to the Discourse of Globalisation. Changing Forms of Knowledge About Change and their Political Consequences. Sociologus 60:41-70.

\section{Autorenangaben:}

PD Dr. Aram Ziai,

Universität Bonn, Zentrum für Entwicklungsforschung,

Walter-Flex-Str. 3, 53113 Bonn,

ziai@uni-bonn.de 